Article

\title{
An Efficient Robust Predictive Control of Main Steam Temperature of Coal-Fired Power Plant
}

\author{
Di Wang, Xiao Wu and Jiong Shen * \\ Key Laboratory of Energy Thermal Conversion and Control of Ministry of Education, Southeast Univerisity, \\ Nanjing 210096, China; wangdi234521@126.com (D.W.); wux@seu.edu.cn (X.W.) \\ * Correspondence: shenj@seu.edu.cn
}

Received: 20 June 2020; Accepted: 21 July 2020; Published: 23 July 2020

\begin{abstract}
Regulating performance of the main steam temperature (MST) system concerns the economy and safety of the coal-fired power plant (CFPP). This paper develops an offset-free offline robust model predictive control (RMPC) strategy for the MST system of CFPP. Zonotope-type uncertain model is utilized as the prediction model in the proposed RMPC design owing to its features of higher accuracy, compactness of representation and less complexity. An offline RMPC aiming at the system robustness and computational efficiency is then developed to maintain the desired steam temperature in case of wide operating condition change. The proposed RMPC is realized by two stages: in the first stage, the RMPC law set, which is the piecewise affine (PWA) of the MST system state is designed offline; then in the second stage, the explicit control law is selected online according to the current state. To achieve an offset-free tracking performance, a manipulated variable target observer is employed to update the chosen RMPC law. The control simulations using on-site operating data of a $1000 \mathrm{MW}$ ultra-supercritical power plant show that the proposed approach can achieve satisfactory control performance and online computation efficiency even under complicated operating conditions.
\end{abstract}

Keywords: energy system control; coal-fired power plant; uncertain model; robust model predictive control; explicit model predictive control

\section{Introduction}

With the rapidly developing of human society and economy, the environmental deterioration arising from fossil fuels firing becomes extremely urgent. However, in China, coal-fired power plants, which are main sources of carbon dioxide and nitrogen dioxide, still produce around $70 \%$ of the whole electricity generation [1], high efficient operation and low carbon emission for coal-fired power plant is fulfilled with large expectation. Consequently, great concern has been paid on the control of main steam temperature (MST), which plays a critical role for the operation of coal-fired power plant (CFPP). The MST must be maintained within an expected range without frequent variation for the economy and safety reasons [2]:

1. The excessively high MST results in serious damage of the superheater and inlet pipe of turbine;

2. The excessively low MST decreases the net efficiency of power plant, and moreover, the steam in the last stage of the low pressure turbine may become wet under low MST condition, which endangers the turbine blades;

3. The frequent temperature fluctuation worsens the heat exchanging in superheater and increases thermal stress of the superheater and turbine cylinder, which will bring material damage to the plant. 
In spite of the importance of MST on the operation of the CFPP, it is still challenging to control the MST due to the large thermal inertia, high nonlinearity and various unpredictable disturbances such as valve vibrations and coal sources variations. Conventional PID-based controllers [3,4], which are robust to deal with the model-mismatches and unpredictable disturbances, can effectively maintain the MST when the CFPP is operated in a given working condition; nonetheless, for a load increase from $50 \%$ working condition to $100 \%$ working condition, the static gain and time constant of the MST control system can increase 6 and 3 times respectively due to the complex process of heat transfer and mass transfer involved in the plant. PID controllers thus cannot achieve satisfactory control performance due to high nonlinearity of the MST system $[5,6]$.

In order to overcome this issue, various control strategies have been developed for the MST regulation. Combined with feedforward compensator, a fuzzy PID control strategy with adjustable proportional coefficient was developed and validated by simulation in [7]. Valsalam et al. [8] used the input-output (I/O) data of MST system to update a Kalman filter, with which the PID controller was redesigned on time, and $\mathrm{N}$-step state prediction of Kalman filter was adopt as the feedback instead of MST. Ma et al. [9] applied an inverse dynamic neuro-control strategy to the MST system in a $300 \mathrm{MW}$ coal-fired power plant. A neuro network (NN) with high accuracy was developed based on the historical I/O data and was used to approximate the inverse dynamic property of MST system. To compensate the mismatches of the NN model, an additional PID controller was developed to guarantee an offset-free control of the MST. An intelligent predictive optimal control method was proposed in [10], the control vector which could make the identified NN model output close to the set point was selected as the control action. However, physical limitations for the valve position of attemperators were not considered in these studies. Therefore, suboptimal control performance can only be achieved.

Model predictive control (MPC) [11,12], which originates from industry practice, is a class of computer control technologies that employs mathematical models to predict future output of the controlled process. At each sampling time, MPC aims to search a control sequence that makes the predictive output follow exactly the exceptive trajectory of the system future output by solving the optimization problem, where the input and state constraints are involved. Owing to the ability of dealing with large inertia characteristics, multivariable coupling and input constraints, MPC has received great concerns form researchers and control engineers. Moreover, the extensibility brings flourishing development of MPC control strategy, which makes it possible to overcome the nonlinearity of controlled process, realize disturbance rejection and guarantee controller robustness. An optimal state estimation MPC method was developed in [13] to reject the unmeasurable disturbance existed in the MST system. A multi-model-based MPC was applied to the MST regulation in [5], where several designed local linear constraint MPCs were switched according to the load demand of power grid. Hlava [14] proposed a nonlinear piecewise affine model-based MPC approach to overcome the nonlinearity produced by wide range load change of the CFPP. To overcome the disturbance due to boiler combustion changing, a multiple disturbance model-based MPC was developed in [15]. In order to meet requirements of MST regulation practice, a nonlinear fuzzy model-based stable MPC approach was presented in [2]. The fuzzy model was developed through combination of several state space type local models estimated by subspace identification approach [16]; and the power output of the unit was selected as the scheduling variable. An offset-free MPC was then designed by introducing disturbance observer and steady-state target calculator, MST regulation simulations demonstrated that issues caused by model mismatches and disturbance occurring were effectively overcome.

Although the effectiveness of these MPC methods was demonstrated by MST system control simulations, none of them have been successfully employed in control engineering practice for the following reasons:

1. Numerical optimization problem must be solved at each sampling interval; the online computational effort of these control algorithms is too heavy; 
2. MST system is operated under complicated working circumstance, such as aging of equipment, complicated combustion process involved in boiler and unpredictable disturbance, however, the robustness of control strategies is rarely involved in the control design.

To resolve the aforementioned difficulties, this paper aims to develop an offline robust MPC approach for the MST system in the CFPP.

Uncertainty sources of the identified model originate from measured data, model structure and parameters. The historical input/output (I/O) data corrupted by unmeasurable noise is used to identify mathematic model, besides, there are unavoidably data gap and abnormal data. Data gap filling methods $[17,18]$ and data fitting methods $[19,20]$ are thus primary concerns for modeling researchers, and fruitful achievements were obtained with persistent efforts of predecessors. Data processing approaches address the problem that identifying data is corrupted by unmeasurable noise and data missing unavoidably exists. Uncertainties of model structure and parameters result from uncertain system identification approach. Compared with Monte Carlo uncertainty analysis [21,22] and Bayesian uncertainty analysis $[23,24]$ which give a probabilistic description of uncertainty, the set-membership identification (SMI) [25-31] aims to achieve a deterministic model uncertainty by bounding the error of uncertain model. There are two kinds of SMI methods according to the uncertain description. One is on the basis of non-parametric model, such as frequency response model $[25,26]$, with which it is challenging to design RMPC controller. The other is parametric model based SMI [27-31], such as ellipsoids, paralellotopes, limited-complexity polyhedrons and zonotopes, among them, zonotope has received much attention in the past decades [29-31] because of the advantages such as high accuracy, good compactness of the representation and low complexity compared with polytopes and ellipsoids. Moreover, zonotope is ideal model for RMPC controller design due to its nature of convex hull. Inspired by zonotope based SMI method [30,31] presented an improved zonotope closed-loop based SMI approach for uncertain system identification. A zonotope-based uncertainty model was achieved in an iterative way via minimizing a new criterion representing the accuracy of the nominal model and the size of uncertainty. Instead of reducing the order after performing the optimization [30], a new optimization problem was built in this paper which aims to circumvent the problem that the order of identified zonotope increases persistently during iteration. The effectiveness was demonstrated by two illustrative examples.

In this paper, an uncertain model is firstly developed by zonotope-based closed-loop SMI approch [31]; and an RMPC is then developed on the zonotope to address the control issues of the MST system, such as high nonlinearity, large inertia, model mismatches and input constraints. The threshold of ideal robust MPC (RMPC) control performance is initially set, the state space is partitioned into several simplex-type subspaces recursively until ideal RMPC control performance of each partitioned subspace is smaller than the threshold, meanwhile, the control laws with guaranteeing robust stability of each subspace vertexes are determined offline by solving a min-max optimization. The local RMPC laws are then linearly combined together to form the global explicit control law online according to the simplex current state belonging to. To drive the MST to track the desired set-point accurately, a manipulated variable target observer is developed based on the nominal model, which is the model with center parameter of the identified zonotope. As a result that control law design is shifted to finish offline, the online computation efficiency of control strategy is improved compared with conventional MPC approach.

The novelties of the proposed MST regulation strategy are summarized as follows,

1. An improved offline RMPC approach is proposed by introducing two extra parameters for a better convergence of the recursive algorithm;

2. A manipulated variable target observer is developed based on the center parameter of zonotope-type prediction model, which can help the RMPC achieve an offset-free control of the MST. 


\section{Main Steam Temperature System}

\subsection{System Description}

The CFPP considered in this paper is a $1000 \mathrm{MW}$ ultra-supercritical power plant located in Shanghai, China. The CFPP is mainly composed by three parts: boiler, turbine and generator. A simplified layout of the power plant is shown in Figure 1. The condensate water preheated by steam extraction from turbine in several heat exchangers is pumped into boiler in which the feed water absorbs the heat from coal combustion and evaporates into steam, after that steam continues to be heated in the superheater system until the temperature increases to the rated degree $\left(600{ }^{\circ} \mathrm{C}\right)$, steam temperature at the outlet is nearly MST; Turbine is an energy convertor where the thermal energy of pressured steam with high temperature is converted into mechanical energy driving the shaft rotating; Generator is equipped on the same shaft of the turbine, in which mechanical energy of the shaft is converted into electric energy.

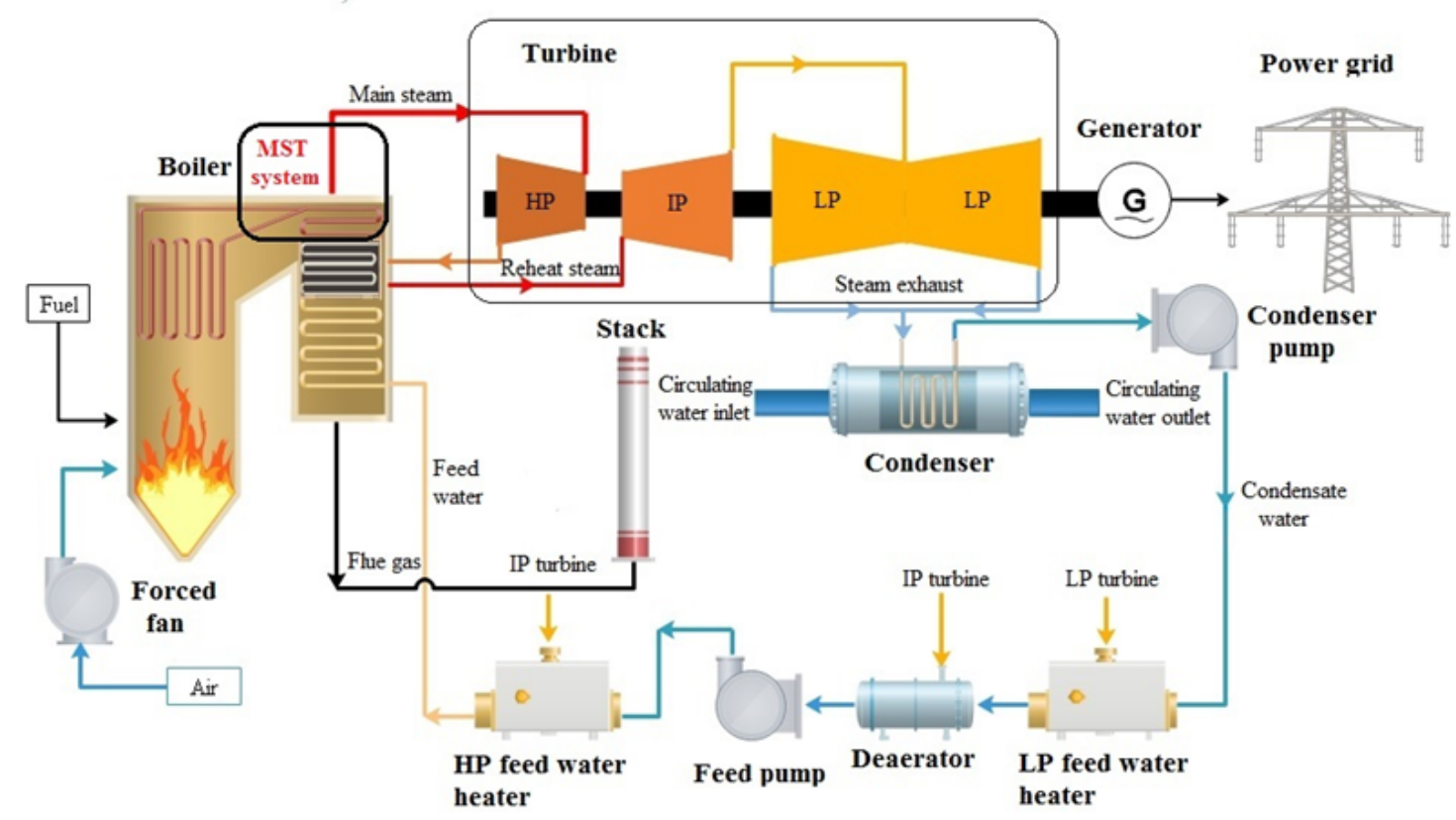

Figure 1. Simplified layout of a $1000 \mathrm{MW}$ coal-fired ultra-supercritical power plant.

The MST system is a crucial subsystem of boiler, whose objective is to maintain MST within an expected range without frequent variation by adjusting the spraying water. The MST system is symmetrically arranged by four superheater subsystems: one primary superheater, one secondary superheater and two attemperators. Figure 2 shows the flowchart of the superheater subsystem considered in this paper. Superheaters are heat exchangers where the pumped steam is heated by absorbing the thermal energy of flue gas produced from combustion of coal and warmed air, and the steam with rated temperature (around $600^{\circ} \mathrm{C}$ ) is delivered to the turbine after passing through the secondary superheater. To guarantee the safety of metal tubes of superheaters and to regulate the steam temperature, purified water is spayed into the steam in primary attemperator and secondary attemperator, the steam is cooled in case of overheat at each superheater inlet. Two valves are utilized to adjust the rate of spray water flow, which are: primary attemperator valve position and secondary attemperator valve position $u$. Generally MST is regulated by $u$, while adjusting primary attemperator valve position is only regarded as a rough regulation to make sure steam temperature of secondary superheater inlet stay in an acceptable range. 


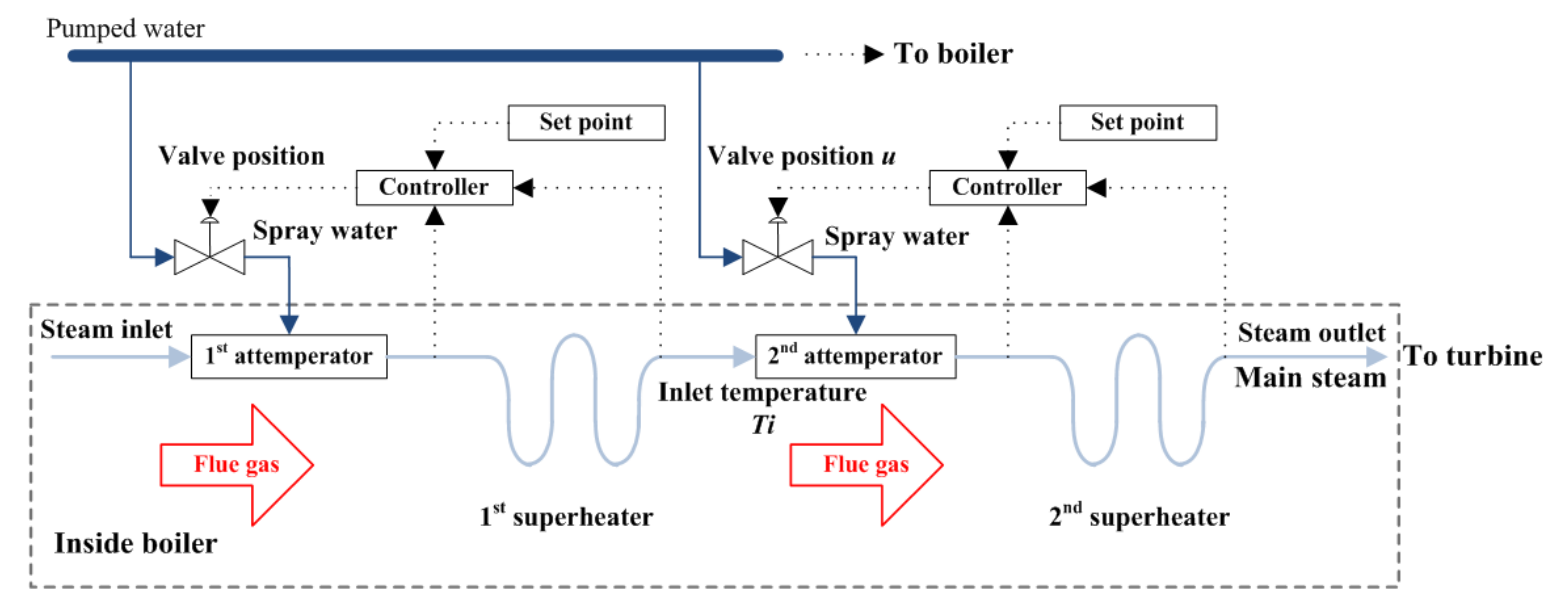

Figure 2. Flowchart of the superheater subsystem.

Figure 3 shows the operating parameters of the MST system when the unit load of CFPP decreases from $820 \mathrm{MW}$ to $450 \mathrm{MW}$. It can be seen that MST appears to have the same variation trend as the steam temperature at the attemperator outlet with a $200 \mathrm{~s}-\mathrm{lag}$, which implies a relatively large thermal inertia property. In addition, MST is influenced directly by steam temperature at attemperator inlet $T i$ (disturbance) and manipulated variable $u$. At high unit load level, there is much more steam passing through the attemperator compared with the situation at low unit load level, thus variation of $u$ makes MST fluctuate less; with unit load demand changing, the heat transfer from flue gas cannot match the changed rate of the steam flow immediately, that causing Ti and MST controlled by inherent PID controller to fluctuate sharply; however, the combustion efficiency gets worse at low unit load level, this issue is essentially unpredictable disturbance occurring, variation of $u$ means a larger change of MST compares with high unit load, a poor control performance is thus achieved. Therefore, advanced control techniques are needed to improve the conventional PID controllers.

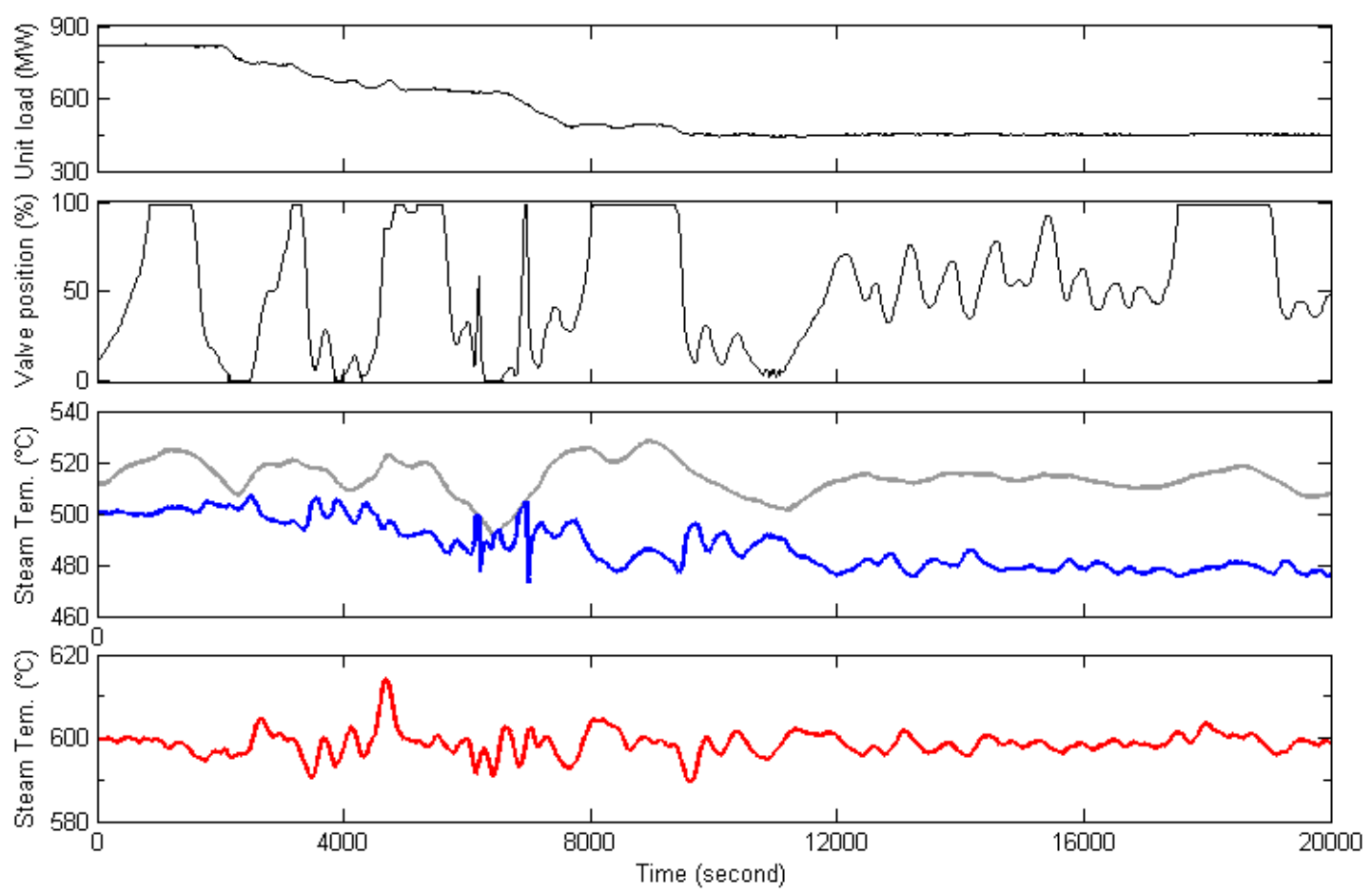

Figure 3. Operating data of MST system during unit load varying (gray line: steam temperature at attemperator inlet $\mathrm{T} i$; red line: MST; blue line: steam temperature at attemperator outlet). 


\subsection{Simulation Model}

According to the properties of MST system discussed above, we can see that the secondary superheater is crucial for MST regulation. Based on fuzzy modeling [2] the nonlinear simulation MST model is established with on-site operating data of the power plant, whose linear local models are identified with I/O operating data of the MST system at unit load demand of $500 \mathrm{MW}, 700 \mathrm{MW}$ and $900 \mathrm{MW}$, respectively. The simulation model output is essentially summation of linearly weighted by the outputs of local models, and the current unit load demand is scheduling variable. Mathematical description of the MST simulation model is shown as follows,

$$
y=f(W, T i, u)
$$

where controlled variable $y \in R$ is MST, manipulated variable $u \in R$ is the valve position of 2nd attemperator, $W \in R$ is unit load, $T i \in R$ is steam temperature at 2 nd superheater inlet which is influenced by unit load variation and unpredictable disturbance such as combustion status change in boiler, $f: R^{3} \rightarrow R$ denotes nonlinear function mapping.

To provide high-fidelity simulation of the MST system, the operating data archived from CFPP is employed as the input $W$ and $T i$ in the simulation model (1). Requirement for MST regulation system designed in this paper is that MST must maintained at rated degree $\left(600^{\circ} \mathrm{C}\right)$ within a wide operating range in cases of unpredictable disturbance and model-mismatch occurring.

\section{Problem Formulation}

RMPC strategy has been proved to have a satisfactory ability in handling the control issues brought by large inertia, nonlinearity and model mismatch while guaranteeing robust stability [32,33].

Consider the following time-variant system with polytope based description,

$$
x_{p}(k+1)=A(k) x_{p}(k)+B(k) u(k),[A(k) \mid B(k)] \in \Omega
$$

where $u(k) \in R^{m}$ and $x_{p}(k) \in R^{n x}$ is the system input and state respectively, $n x$ and $m$ are positive integers, $A(k) \in R^{n x \times n x}$ and $B(k) \in R^{n x \times m}$ are time-varying parameter matrices which belong to convex hull model set $\Omega$,

$$
\Omega=\left\{[A(k) \mid B(k)] \mid \sum_{j=1}^{h} \omega_{j}(k)=1, \omega_{j}(k) \geq 0,[A(k) \mid B(k)]=\sum_{j=1}^{h} \omega_{j}(k)\left[A_{j} \mid B_{j}\right]\right\}
$$

where $\left[A_{j} \mid B_{j}\right]\left(A_{j} \in R^{n x \times n x}, B_{j} \in R^{n x \times m}, j \in\{1, \cdots, h\}\right)$ is the vertex of the convex hull $\Omega, \omega_{j}(k)$ is weighting coefficient. For MST system, valve position of attemperator $u$ has physical restriction such that the following input constraint must be posed,

$$
\underline{u} \leq u(k+i) \leq \bar{u}
$$

where $\bar{u}=\left[\bar{u}_{1}, \cdots, \bar{u}_{m}\right]^{T}$ is positive column vector, and $\underline{u}=\left[\underline{u}_{1}, \cdots, \underline{u}_{m}\right]^{T}$ negative column vector, superscript " $T$ " denotes matrix transposition.

The following state feedback and infinite-horizon performance index are adopted,

$$
\begin{gathered}
u(k+i \mid k)=K x_{p}(k+i \mid k), \forall i \geq 0 \\
J_{\infty}\left(x_{p}(k)\right)=\sum_{i=0}^{\infty}\left\|x_{p}(k+i \mid k)-x_{s}\right\|_{L_{x}}^{2}+\left\|u(k+i \mid k)-u_{s}\right\|_{L_{u}}^{2}
\end{gathered}
$$

where $K \in R^{m \times n x}$ is the state feedback matrix, $\left\|x_{p}(k+i \mid k)\right\|_{L_{x}}^{2}=x_{p}(k+i \mid k)^{T} L_{x} x_{p}(k+i \mid k)$, whose operation is the same as $\|u(k+i \mid k)\|_{L_{u^{\prime}}}^{2} L_{x} \in R^{n x \times n x}>0$ and $L_{u} \in R^{m \times m}>0$ are symmetric 
positive weighting matrices. Thus, the worst case of the performance index (6) is utilized as robust control cost in this section,

$$
\max _{\{A(k+j) \mid B(k+j)\} \in \Omega, j \geq 0} \sum_{i=0}^{\infty}\left\|x_{p}(k+i \mid k)-x_{s}\right\|_{L_{x}}^{2}+\left\|u(k+i \mid k)-u_{s}\right\|_{L_{u^{\prime}}}^{2} \text { s.t. }(2)
$$

Define Lyapunov function $V\left(x_{p}\right)=x_{p}^{T} P x_{p}$, the inequality of matrix $P>0$ means $P$ is symmetric positive matrix, and the following formulation must be held,

$$
V\left(x_{p}(k+i+1 \mid k)\right)-V\left(x_{p}(k+i \mid k)\right) \leq-\left\|x_{p}(k+i \mid k)\right\|_{L_{x}}^{2}-\|u(k+i \mid k)\|_{L_{u}}^{2}
$$

Summing (8) from $i=1$ to $i=\infty$, obtain the upper bound $J_{\infty}\left(x_{p}(k)\right) \leq V\left(x_{p}(k \mid k)\right)$.

Theorem 1 ([32]). Let $x(k \mid k)$ be the state of the controlled system measured at sampling time $k$, the uncertain model set is polytope based description defined as (3). Then the state feedback matrix $\mathrm{K}$ in the control law (5) that minimizes the upper bound on the robust performance objective function at sampling time $k$ and satisfies a set of specified input is given by

$$
K=Y Q^{-1}
$$

where the symmetric positive matrix $Q \in R^{n x \times n x}>0$ and matrix $Y \in R^{m \times n x}$ are obtained from the solution of the following linear objective minimization problem with LMI-type constraints:

$$
\min _{Y, Q} \gamma
$$

where $\gamma$ is positive scalar to be optimized with the relationship $P=\gamma Q^{-1}$. The receding-horizon implementation of the RMPC algorithm in (10) guarantees exponential closed-loop stability, once a feasible solution of the MPC problem is found.

$$
\begin{aligned}
& {\left[\begin{array}{cc}
1 & * \\
x(k \mid k) & Q
\end{array}\right] \geq 0} \\
& {\left[\begin{array}{cccc}
Q & * & * & * \\
A_{j} Q+B_{j} Y & Q & * & * \\
L_{x}^{1 / 2} Q & 0 & \lambda I & * \\
L_{u}^{1 / 2} Y & 0 & 0 & \lambda I
\end{array}\right] \geq 0, j \in\{1, \cdots, h\}} \\
& {\left[\begin{array}{cc}
Z & Y \\
Y^{T} & Q
\end{array}\right] \geq 0, Z \leq(\min \{\bar{u},-\underline{u}\})^{2}}
\end{aligned}
$$

The recursive feasibility is demonstrated in [32].

The optimal control input $u^{*}\left(x_{p}(k)\right)=K^{*}\left(x_{p}(k)\right) x_{p}(k)$ is calculated by solving the LMIs (10) at each control interval, which aims to drive the system (2) to the steady state $\left(x_{s}, u_{s}\right)$. However, the online computation burden is too heavy for control practice, approximated explicit MPC (AEMPC) [34] is thus proposed, whose basic idea is to achieve a suboptimal solution of (10) instead via partitioning polytope-type initial state space $\Phi_{0}$ into $n$ subspaces $\left\{S_{p k}\right\},(k=1,2, \cdots, n)$ and the obtained approximated control input in piece-wise affine (PWA) explicit form is shown as Formulation (12).

$$
\begin{aligned}
& \hat{u}\left(x_{p}(k)\right)=H_{i} x_{p}(k)+b_{i}, \forall x_{p}(k) \in S_{p k} \\
& \bigcup_{k=1}^{n} S_{p k}=\Phi_{0}, \Phi_{0} \triangleq\left\{x \in R^{n x} \mid D x \leq b\right\}
\end{aligned}
$$


Compared with implicit control law achieved by conventional MPC, the explicit control law (12) tends to be clearly accepted by control engineers. In addition, AEPMC is essentially an offline control approach, which achieves enhanced online computation efficiency. However, standard AEMPC algorithm has poor convergence for RMPC controller design, and classical explicit MPC [34-36] cannot deal with target tracking problem.

\section{An Improved Offline Robust Model Predictive Control Approach for MST System}

To overcome the drawbacks of AEMPC, an approximated explicit RMPC (OFAERMPC) method is presented in this section, which is realized in two stages: the offline stage and online stage, the control structure is shown as Figure 4. In the offline stage, an improved state space partition strategy is developed to strengthen the convergence by introducing two parameters avoiding the recursive algorithm falling into local endless loop. The whole state space is partitioned into a great number of simplex-type subspaces and control laws corresponding to the subspaces are then designed based on the RMPC approach stated in previous subsection. In the online stage, at each control interval the explicit RMPC control law is searched according to the current state, and the targeted control input is updated based on the nominal model of uncertain system identified by the SMI approach [31], furthermore, the input variation restriction is imposed on the explicit RMPC control law.

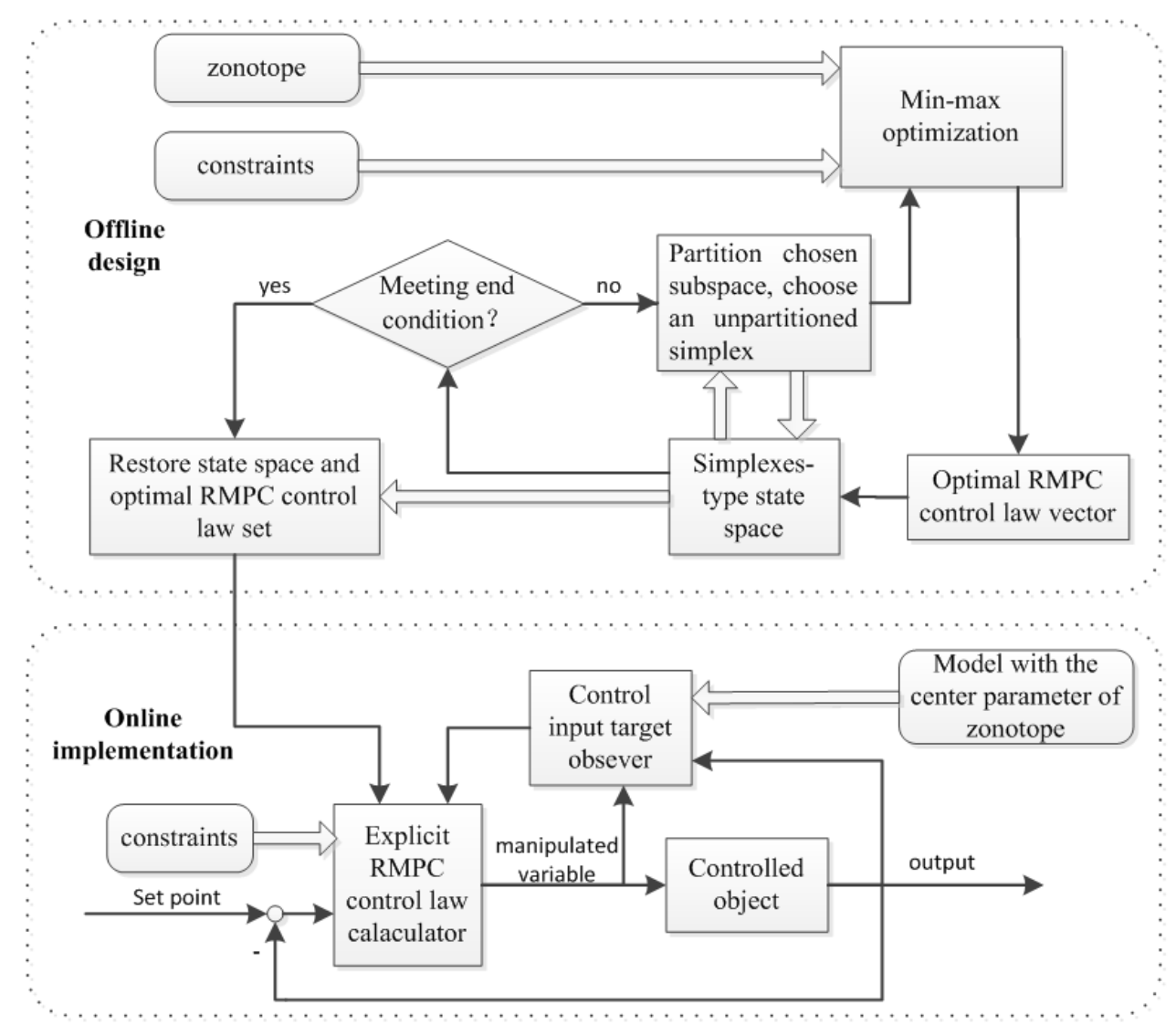

Figure 4. The schematic of an approximated explicit robust model predictive control (OFAERMPC).

\subsection{The Offline Design for RMPC Control Law}

A simplex $S_{p}$ in $n x$-dimensional space is described mathematically as,

$$
S_{p} \triangleq\left\{x_{p} \mid \sum_{j=1}^{n x+1} \omega_{j}(k)=1, x_{p}=\sum_{j=1}^{n x+1} \omega_{j}(k) x_{p j}, \omega_{j}(k) \geq 0, x_{p j} \in R^{n x}\right\}
$$


where $\left\{x_{p j}(j=1, \cdots, n x+1)\right\}$ are the $n x+1$ vertexes of simplex $S_{p}$.

As shown in (13), a simplex in n-dimensional space is a subspace surrounded by envelope lines composed by $n x+1$ points. Simplex is fundamental component of geometries in multi-dimensional space, a 1-dimensional simplex is an interval, a 2-dimensional simplex is a triangle and a 3-dimensional simplex is a tetrahedron. As a result of its convexity, any point in simplex can be represented linearly by the simplex vertexes.

An extended state matrix $M$ of the simplex $S_{p}$ is defined as $M \triangleq\left[\begin{array}{ccc}1 & \ldots & 1 \\ x_{p 1} & \ldots & x_{p(n x+1)}\end{array}\right] \in$ $R^{(n x+1) \times(n x+1)}$, thus, for any point $x_{p} \in S_{p}$, the following relationship is held,

$$
M^{-1}\left[\begin{array}{ll}
1 & x_{p}
\end{array}\right]^{T}=\left[\begin{array}{lll}
\omega_{1}(k) & \cdots & \omega_{n x+1}(k)
\end{array}\right]^{T}
$$

Define optimal robust predictive control vector of the simplex $S_{p}$ as

$$
U \triangleq\left[\begin{array}{lll}
u^{*}\left(x_{p 1}\right) & \cdots & u^{*}\left(x_{p(n x+1)}\right)
\end{array}\right]
$$

where $u^{*}\left(x_{p j}\right)(j=1, \cdots, n x+1)$ is the robust predictive control input calculated by solving (10) with $x_{p j}$ being initial system state, and the corresponding optimal robust cost index vector $V V$ is defined as,

$$
V V \triangleq\left[\begin{array}{lll}
V^{*}\left(x_{p 1}\right) & \cdots & V^{*}\left(x_{p(n+1)}\right)
\end{array}\right]^{T}
$$

For any point $x_{p} \in S_{p}$, define approximated optimal robust predictive control input as linearly weighted summation of the optimal one of simplex vertexes,

$$
\hat{u}\left(x_{p}\right) \triangleq U M^{-1}\left[\begin{array}{cc}
1 & x_{p}
\end{array}\right]^{T}
$$

Approximated optimal robust cost index $\bar{V}\left(x_{p}\right)$ defined as (18) is utilized.

$$
\bar{V}\left(x_{p}\right) \triangleq V V M^{-1}\left[\begin{array}{ll}
1 & x_{p}
\end{array}\right]^{T}
$$

It is expected that the explicit subspace approximated optimal control input $\hat{u}\left(x_{p}\right)$ with explicit PWA form is used in prescribed state instead of optimizing (10) online to achieve $u^{*}\left(x_{p}\right)$. To this end, the situation that the smaller volume of the simplex makes $\bar{V}\left(x_{p}\right)$ closer to $V^{*}\left(x_{p}\right)$ can be regarded as the guide for state space partitioning.

Define approximated robust cost index deviation as $\xi\left(x_{p}\right) \triangleq \bar{V}\left(x_{p}\right)-V^{*}\left(x_{p}\right)$, of which the threshold $\sigma$ is set, for any point $x_{p} \in S_{p}$, let $\xi\left(x_{p \max }\right)=\max _{x_{p} \in S} \xi\left(x_{p}\right)$, the state point $x_{p \max }$ is obtained by solving the following optimization problem.

$$
\begin{aligned}
& \max _{\gamma, Y, Q, x_{p \max }} \xi_{a}\left(x_{p \max }\right)=V M^{-1}\left[\begin{array}{ll}
1 & x_{p \max }
\end{array}\right]^{T}-\gamma \\
& \text { s.t. }(11), M^{-1}\left[\begin{array}{lll}
1 & x_{p \max }
\end{array}\right]^{T} \geq 0
\end{aligned}
$$

If $\xi\left(x_{p \max }\right) \leq \sigma$, then this simplex $S_{p}$ is well partitioned, and next simplex is chosen; if $\xi\left(x_{p \max }\right)>\sigma$, it means that chosen simplex $S_{p}$ need to be partitioned into $n x+1$ simplexes that the $n x+1$ simplex vertexes are replaced by the state point $x_{p \max }$ in sequence.

An offline recursive control law design with enhanced convergence is proposed in this section, which aims at partitioning polyhedral initial state space $\Phi_{0}$ into simplex-type subspaces and designing RMPC control laws of the corresponding simplexes that drive the system to the origin. The procedure is given as follows: 
1. Partition $\Phi_{0}$ into several simplexes $S_{i}, i \in\{1,2, \cdots, l\}$ applying Delaunay triangulation [37], i.e., $\Phi_{0}=\left\{S_{1}, \cdots, S_{l}\right\}$, calculate and store respectively $V V_{i} \triangleq\left[\begin{array}{lll}V^{*}\left(x_{p 1}\right) & \cdots & V^{*}\left(x_{p(n x+1)}\right)\end{array}\right]^{T}$ and $U_{i} \triangleq\left[\begin{array}{lll}u^{*}\left(x_{p 1}\right) & \cdots & u^{*}\left(x_{p(n x+1)}\right)\end{array}\right]^{T}$ at simplexes vertexes state points via (10), build $M_{i}$, preset threshold $\sigma, \alpha$ and $\beta$, let $i=1$;

2. If $i \leq l$, select the current simplex $S_{i}$ and turn to Step 3; if $i>l$, algorithm ends, return $U \triangleq\left[\begin{array}{lll}U_{1} & \cdots & U_{h}\end{array}\right]$ and $M \triangleq\left[\begin{array}{lll}M_{1} & \cdots & M_{h}\end{array}\right], h$ is size of well-partitioned space $\Phi_{0} ;$

3. Compare the size of $\alpha$ and conditional number of $M_{i}$, if $\operatorname{cond}\left(M_{i}\right)>\alpha$, delete $S_{i}, l=l-1$, and turn to Step 2; if cond $\left(M_{i}\right) \leq \alpha$, turn to Step 4;

4. Obtain $\max _{x_{p \max } \in S} \xi_{a}\left(x_{p \max }\right), x_{p \max }, V^{*}\left(x_{p \max }\right)$ and $u^{*}\left(x_{p \max }\right)$ via (19), if $\max _{x_{p \max } \in S} \xi_{a}\left(x_{p \max }\right) \leq \sigma$, $i=i+1$, and turn to Step 2; if $\max _{x_{\max } \in S} \xi_{a}\left(x_{p \max }\right)>\sigma$, replace the $n x+1$ vertexes of $S_{i}$ with $x_{p \max }$ in sequence yielding $n x+1$ new simplex, add them to $\Phi_{0}$, and delete $S_{i}, l=l+n x$, turn to Step 5;

5. Determine whether the longest side is $\beta$ times longer than the shortest side, if not, turn to Step 2; if yes, take the midpoint of the longest side as a new point $x_{p z}$, calculate $V^{*}\left(x_{p z}\right)$ and $u^{*}\left(x_{p z}\right)$, two end points of the longest side are replaced with $x_{p z}$ in sequence, then two simplexes yield, delete $S_{i}, l=l+1$, turn to Step 2 .

Remark 1. This proposed algorithm of offline design for RMPC control is developed on the AEMPC. However, when applying AEMPC to design offline RMPC method the convergence problem arises, the reality that the optimal solution $x_{p \max }$ of (19) in current simplex $S_{i}$ approaches closely one of vertexes or the longest side of $S_{i}$ results in partitioning current simplex too many times, falling into endless loop can even occur. Therefore, the proposed method introduces two parameters $\alpha$ and $\beta$ to accelerate convergence rate. Another disadvantage is that AEMPC cannot effectively deal with control target tracking problem for MST system, because during MST system normal operation the control input target is generally unknown owing the nonlinearity of dynamic property and unmeasurable disturbance existing, the relevant solution will be proposed in following subsection.

Remark 2. If $\sigma$ decreases, then the approximated optimal control input $\hat{u}\left(x_{p}\right)$ is closer to the optimal one, but the size of partitioned simplexes and the calculation offline burden become larger, it is recommended that both criterions $\max _{x_{p \max } \in S_{i}} \xi_{a}\left(x_{p \max }\right) / V^{*}\left(x_{p \max }\right)<\sigma_{r}$ and $\max _{x_{p \max } \in S_{i}} \xi_{a}\left(x_{p \max }\right)<\sigma_{a}$ are adopted, i.e., the current simplex is considered to be appropriate as long as either of conditions is true; if $\alpha$ increases, then offline computational burden will reduce, and the possibility of extended state matrix $M$ being illness will larger and the volume of the simplex $S_{i}$ will decrease accordingly, causing $x_{p \max }$ to be too closer to one of the vertexes, which must yield a worse convergence; if $\beta$ increases, then offline computational burden will reduce, and the point $x_{p \max }$ will tend to approach the longest side of the simplex $S_{i}$, which must yield a convergence problem.

The designed offline control law is $(M, U)$, in which $M$ and $U$ illustrate the well partitioned subspace and control law set respectively. In addition, according to (17), we see that the approximated RMPC control law $\hat{u}\left(x_{p}(k)\right)$ essentially has an explicit PWA formulation with respect to the current state $x_{p}(k)$.

\subsection{The Online Implementation for Offline Designed RMPC Control Law}

The basic requirement of the MST regulation system is to maintain the constantly at the set point; however, the control input target is actually unknown and varies frequently during the routine operation, which will deliver the control result with offset when applying offline RMPC approach [34-36]. On the other hand, to alleviate wear and tear of the attemperator valve, a control input variation rate restriction must be involved in controller design. For these reasons, an offset-free online implementation of offline RMPC with input amplitude and variation rate constraints is developed in this subsection. 
To this end, control of MST system is viewed as target tracking with controlled state set point $x_{r}(k)$ and unknown control input set point $u_{r}(k)$, which can be solved by driving the system to track the control target $\left(x_{r}(k), u_{r}(k)\right)$. On the basis of PWA-type RMPC control law designed in Section 4.1, the offset-free control input with unknown control input set point $u_{r}(k)$ is shown as follows,

$$
u(k)=U_{i} M_{i}^{-1}\left[\begin{array}{cc}
1 & x_{c}(k)-x_{r}(k)
\end{array}\right]^{T}+u_{r}(k)
$$

where $x_{\mathcal{c}}(k) \in R^{n x \times n x}$ is the measured system state at time instant $k$, the matrix $U_{i} \in R^{m \times(n x+1)}$ and $M_{i} \in R^{(n x+1) \times(n x+1)} i \in\{1,2, \cdots, h\}$ are obtained by searching in the $U$ and $M$ with tracking error $x_{c}(k)-x_{r}(k)$ at time instant $k, h$ is the size of well-partitioned subspaces.

With control law (20), an offset-free control strategy can be achieved by estimating the unknown control input set-point $u_{r}(k)$ online according to historical I/O data. Consider the nominal model identified by zonotope based closed-loop set-membership identification method [31], and let $x_{0}(k-1)=x_{c}(k-1)$ at time instant $k-1$, we have

$$
x_{0}(k)-x_{r}(k)=A_{0}\left(x_{c}(k-1)-x_{r}(k-1)\right)+B_{0}\left(u(k-1)-u_{r}(k-1)\right)
$$

where the parameter matrices $A_{0}(k)$ and $B_{0}(k)$ are the model with center parameter of zonotope-type uncertain model.

Given that there exists a control input $u_{b}(k-1)$ holding the following relation,

$$
x_{c}(k)-x_{r}(k)=A_{0}\left(x_{c}(k-1)-x_{r}(k-1)\right)+B_{0} u_{b}(k-1)
$$

We have $u_{b}(k-1)=B_{0}^{+}\left(x_{p}(k)-x_{r}(k)-A_{0}\left(x_{p}(k-1)-x_{r}(k)\right)\right)$, the superscript " + " is pseudo inverse operator. (22) subtracts (23), we have the nominal state error,

$$
x_{c}(k)-x_{0}(k)=B_{0}\left(u_{b}(k)-u(k-1)+u_{r}(k-1)\right)
$$

For the nominal system (21), let $u_{r}(k-1)$ be the estimation of unknown $u_{r}(k)$,

$$
x_{0}(k+1)-x_{r}(k+1)=A_{0}\left(x_{c}(k)-x_{r}(k)\right)+B_{0}\left(u(k)-u_{r}(k-1)\right)
$$

Since $u_{r}(k)$ is unknown at the current instant $k$, the nominal system prediction output $x_{0}(k+1)$ must be biased from the target $x_{r}(k+1)$. To alleviate this issue, historical nominal state error $x_{c}(k)-x_{0}(k)$ is used to compensate the future nominal state value in (24), i.e., both left and right side of Formulation (23) times a compensation coefficient $a(0 \leq a \leq 1)$ and then sum (24), we have,

$$
\begin{aligned}
& x_{0}(k+1)-x_{r}(k+1)+a\left(x_{c}(k)-x_{0}(k)\right) \\
& =A_{0}\left(x_{c}(k)-x_{r}(k+1)\right)+B_{0}\left(u(k)-\left(a\left(u(k-1)-u_{b}(k)\right)+(1-a) u_{r}(k-1)\right)\right)
\end{aligned}
$$

According to (25), a control input target observer is thus obtained as $\widehat{u}_{r}(k)=a\left(u(k-1)-u_{b}(k)\right)+$ $(1-a) u_{r}(k-1)$.

Therefore, the control action at time instant $k$ will be imposed on the controlled system shown as (26),

$$
u(k)=\left\{\begin{array}{c}
u(k-1)+(1-a)\left(u_{r}(k-1)-u(k-1)\right)+U_{i} M_{i}^{-1}\left[\begin{array}{cc}
1 & x_{c}(k)-x_{r}(k)
\end{array}\right]^{T}- \\
a B_{0}^{+}\left(x_{c}(k)-x_{r}(k)\right)+a B_{0}^{+} A_{0}\left(x_{c}(k-1)-x_{r}(k-1)\right),\left(x_{c}(k)-x_{r}(k)\right) \in \Phi_{0} \\
u(k-1),\left(x_{c}(k)-x_{r}(k)\right) \notin \Phi_{0}
\end{array}\right.
$$

where $\Phi_{0}$ is the well-partitioned state space in the offline design stage. 
Remark 3. As shown in (26), at time instant $k$ the final explicit control input has the same mathematical representation as the one of digital PID controller, in (26) a $B_{0}^{+}\left(A_{0}\left(x_{c}(k)-x_{r}(k)\right)-\left(x_{c}(k)-x_{r}(k)\right)\right)+$ $U_{i} M_{i}^{-1}\left[1 \quad x_{c}(k)-x_{r}(k)\right]^{T}$ illustrates "Proportion" action and $a B_{0}^{+}\left(A_{0}\left(x_{c}(k-1)-x_{r}(k-1)\right)-\right.$ $\left.\left(x_{c}(k)-x_{r}(k)\right)\right)$ illustrates "Integration" action. We see that the final control input (26) becomes essentially nonlinear PI controller by introducing a control input target observer, since the integral action with respect to the tracking error is included in the control input, the offset-free tracking is guaranteed with the proposed approach.

As for MST system, the control input variation rate restriction must be imposed in case of the wear and tear of the attemperator valve.

$$
\underline{d u} \leq u(k)-u(k-1) \leq \overline{d u}
$$

where $\overline{d u}$ and $d u$ is upper and lower bound of control input variation rate.

\section{Simulation Results}

The proposed offline RMPC approach for MST system is tested by control simulations in this section. A zonotope-type uncertain model for controller design is achieved firstly in a recursive way, and then proposed control strategy for MST system is demonstrated via comparison with PID and other MPC methods.

\subsection{Establishment of the Zonotope-Type Uncertain Model for MST System}

In this section, on-site data of an ultra-supercritical CFPP located in in Shanghai, China is recorded to establish the prediction model for controller design through a closed-loop identification method for uncertain system, and the principles of algorithm realization are referred to reference [31]. Figure 5 shows 3000 groups of routine operating data of MST system around unit load $700 \mathrm{MW}$ with sampling interval of $5 \mathrm{~s}$. The last 2000 groups of I/O data are utilized to identify uncertain model for MST system and the first 1000 groups of $\mathrm{I} / \mathrm{O}$ data are used as verification data set to demonstrate the effectiveness of the identified model.

The model structure, which is compatible with identification data set, can be achieved by the asymptotic method [38], i.e., $G\left(z^{-1}\right)=\frac{b_{1} z^{-1}}{1+a_{1} z^{-1}}$, there are two parameters to be identified, and the order of zonotope is 3 , thus, the zonotope-type uncertain model at time instant $k$ is $\operatorname{AFSS}(k)=\left\{\theta=\left[\begin{array}{ll}a_{1} & b_{1}\end{array}\right]^{T} \mid \theta \in c \oplus H B^{3}\right\}$, in which $B^{3}$ means an 3-order unitary box, let the model with center parameter $c=\left[\begin{array}{ll}a_{0} & b_{0}\end{array}\right]^{T}$ be the nominal model of $\operatorname{AFSS}(k)$, and $H B^{3}$ illustrates variation range of the parameters $a_{0}$ and $b_{0}$. Initial nominal parameter vector can be estimated via the output error identification method [39], and $H B^{3}$ must be set large enough to cover the true parameter, to this end, the initial zonotope-type uncertain model is shown as (28).

$$
\operatorname{AFSS}(0)=\left[\begin{array}{l}
-0.978 \\
-0.005
\end{array}\right] \oplus\left[\begin{array}{ccc}
0.02 & 0 & 0 \\
0 & 0.01 & 0
\end{array}\right] B^{3}
$$

where the notion " $\oplus$ " denotes the Minkowski summation of two sets $X_{1}$ and $X_{2}$, i.e., $X_{1} \oplus X_{2}=$ $\left\{x \mid x_{1}+x_{2}, x_{1} \in X_{1}, x_{2} \in X_{2}\right\}$. 

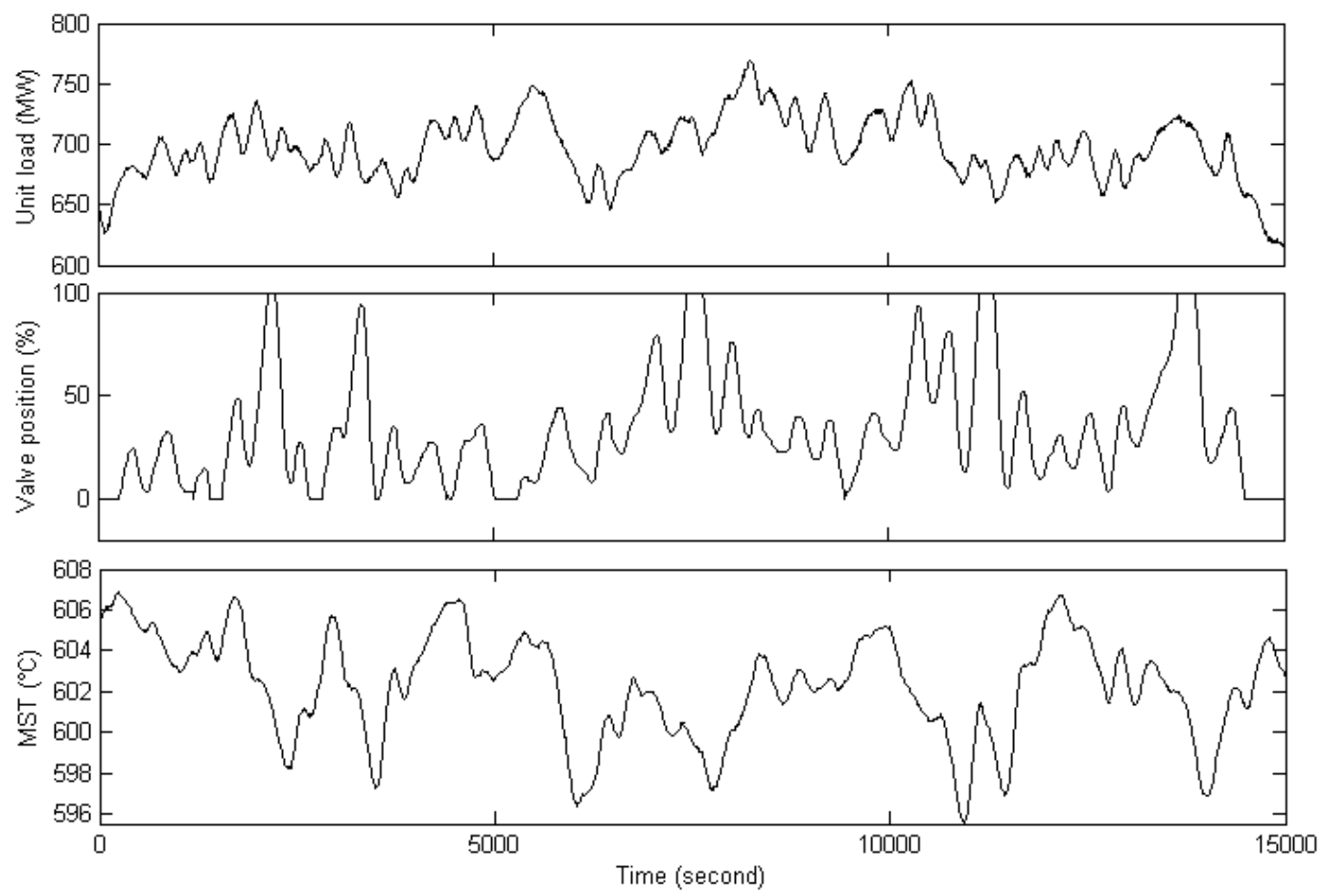

Figure 5. Routine operating data for main steam temperature (MST) system identification and verification.

Essential parameters of closed-loop identification method [31] are set in Table 1.

Table 1. Parameters of the identification algorithm.

\begin{tabular}{cc}
\hline Order of pre-estimation model & 8 \\
Error bound to be restrained $\Gamma$ & 1.76 \\
Weighting ratio $w_{H} / w_{e}$ & 50 \\
\hline
\end{tabular}

The final uncertain model is achieved by applying the recursive closed-loop identification method [31] shown as (29).

$$
\operatorname{AFSS}(2000)=\left[\begin{array}{l}
-0.9866 \\
-0.0018
\end{array}\right] \oplus\left[\begin{array}{ccc}
0.0029 & -6.41 \times 10^{-7} & 0 \\
0.0012 & 0 & -1.55 \times 10^{-5}
\end{array}\right] B^{3}
$$

where $v_{i},(i=1,2, \cdots, 6)$ are vertexes of the convex hull listed as Table 2.

Table 2. Vertexes of identified model (30).

\begin{tabular}{cccccc}
\hline $\boldsymbol{v}_{\mathbf{1}}$ & $\boldsymbol{v}_{\mathbf{2}}$ & $\boldsymbol{v}_{\mathbf{3}}$ & $\boldsymbol{v}_{\mathbf{4}}$ & $\boldsymbol{v}_{\mathbf{5}}$ & $\boldsymbol{v}_{\mathbf{6}}$ \\
\hline-0.989561 & -0.983721 & -0.989562 & -0.983721 & -0.983722 & -0.989562 \\
-0.002991 & -0.000593 & -0.002991 & -0.000562 & -0.000562 & -0.002960 \\
\hline
\end{tabular}

Model validation is conducted via the verification data set, the result is shown as Figure 6. We see that the nominal output and the output bound of the uncertain model has the similar variation trend to the one of the real output. However, the difference from the ideal simulation experiment is that the output of uncertain model identified with the on-site operating I/O data cannot cover the whole real output, because complicated uncertain disturbances unavoidably influence MST system, there is information deviation between identification data set and verification data set, moreover, in the identification and verification period MST system show different nonlinearity. Nonetheless, compared 
with the model with sole parameter, parameters of the uncertain model belong to specific model set causes the output to cover the real output, which illustrates the uncertain model has improved descriptive ability for MST system.
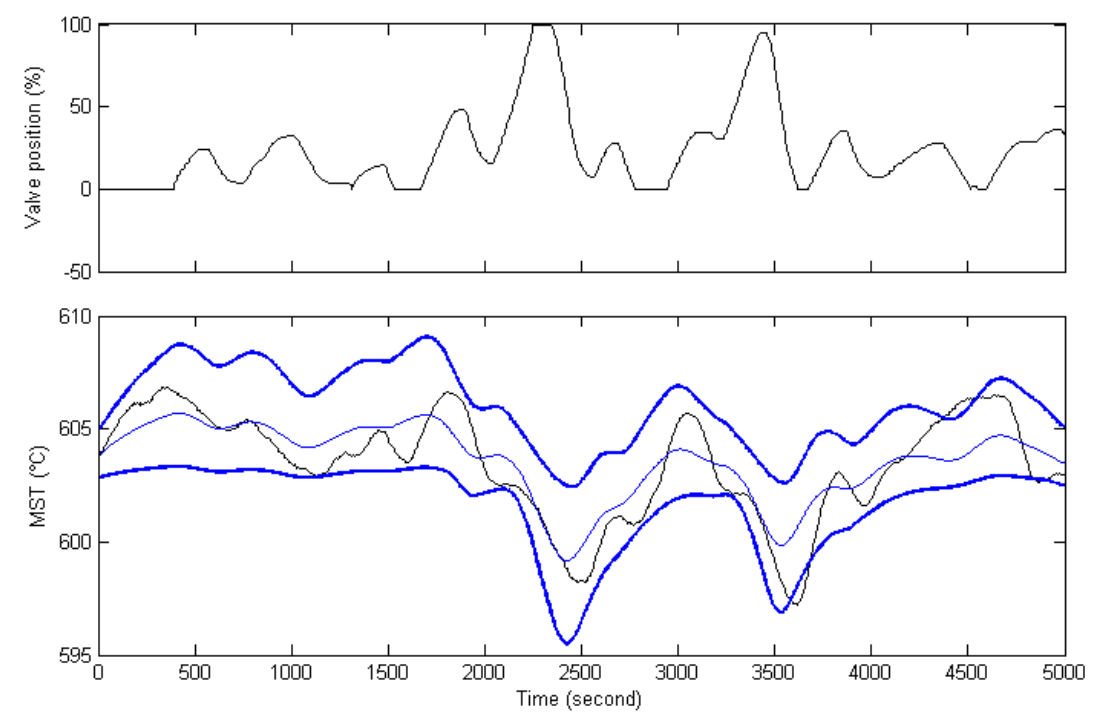

Figure 6. Verification of identified uncertain model (blue thin line: identified nominal model output; blue heavy line: identified uncertain model output bounds; black line: real output).

Step response test of the identified uncertain model (44) is shown as Figure 7, the upper part of Figure 7 illustrates the valve position (input of model (44)) variation, and the lower part illustrates the MST (output of model (44)) variation with the valve position varying. At $t=50 \mathrm{~s}$, the valve position steps from $47.73 \%$ to $52.73 \%$, the output decreases slowly from $600{ }^{\circ} \mathrm{C}$, the nominal model output approaches to $599.3{ }^{\circ} \mathrm{C}$ at $t=1600 \mathrm{~s}$, while the uncertain output is a range between $598.7^{\circ} \mathrm{C}$ and $599.7^{\circ} \mathrm{C}$ with a transient time of $1400-2000 \mathrm{~s}$, the dynamic characteristics shows with a large thermal inertia. We can see that the identified uncertain model with a varying static gain and transient time is essentially nonlinear, with which it is effective to describe the dynamic features of MST system that the static gain and transient time will keep changing with the unit load following the command from the power grids.
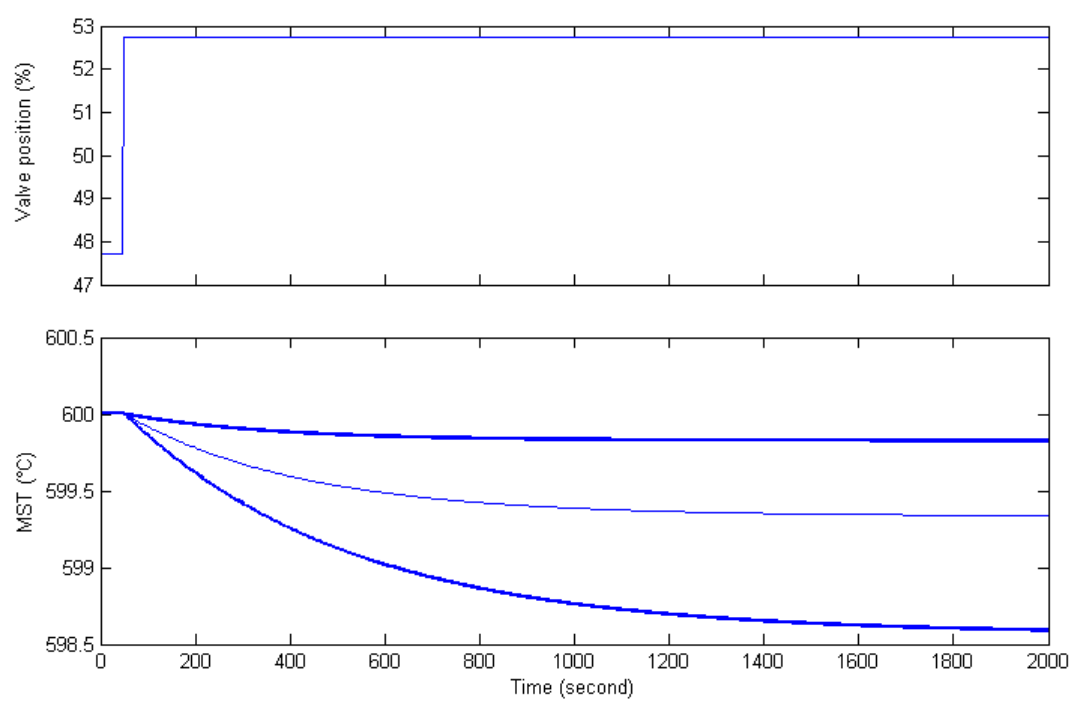

Figure 7. Step test of identified uncertain model (blue thin line: identified nominal model output; blue heavy line: identified uncertain model output bounds). 


\subsection{Control Simulation for MST System}

In this section, the proposed OFAERMPC method is utilized to design controller for MST system of $1000 \mathrm{MW}$ ultra-supercritical power plant, the control objective is that MST is kept at $600{ }^{\circ} \mathrm{C}$ with less fluctuation under complicated operating conditions while guaranteeing the stability by adjusting the valve position of 2 nd attemperator.

Simulation platform: matlab2015b with yalmip solver [40]; computer hardware: CPU—Intel Core i5-2410M, RAM-DDR3 6 GB.

The corresponding parameters of OFAERMPC and standard AEMPC are set as follows: initial state space $\Phi_{0}=\{x \in R \mid-15 \leq x \leq 15\} ; \sigma=0.005, \alpha=3, \beta=1000$; weighting coefficients $L_{x}=1$ and $L_{u}=0.01$; compensation coefficient $a=0.1$; sampling interval $T s=5 \mathrm{~s}$; amplitude and variation rate constraints of manipulated variable are $0 \leq u(k) \leq 100 \%$ and $-4 \% \leq u(k)-u(k-1) \leq 4 \%$. The offline computation time and number of partitioned subspaces are listed in Table 3.

Table 3. Offline performance of OFAERMPC and standard approximated explicit model predictive control (AEMPC).

\begin{tabular}{ccc}
\hline & Computation Time & Number of Subspaces \\
\hline Standard AEMPC & $3619 \mathrm{~s}$ & 1914 \\
OFAERMPC & $170 \mathrm{~s}$ & 108 \\
\hline
\end{tabular}

In the offline control law design stage, OFAERMPC consumes much less time than standard AEMPC, and obtains a state space partition with fewer subspaces. We can see that standard AEMPC achieves a poor convergence when designing MST controller, which is actually an SISO system. However, when applying the basic idea of AEMPC to design RMPC controller for the MIMO system, the control algorithm may suffer from falling into endless loop.

Three case studies are considered to demonstrate the effectiveness of the proposed OFAERMPC method.

\section{Case 1. Power plant unit load varies}

During the routine operation of power plant, to hold the power grid frequency constant the unit load must trace the load demand from the grid, therefore, the unit load daily varies according to the demand of electricity users. However, the steam temperature of attemperator inlet $T i$ and dynamic property of MST system will change correspondingly when the unit load $W$ varies, the valve position of attemperator $u$ must be regulated to hold MST constant with less fluctuation. Objective of this control simulation is to test control performance of the proposed OFAERMPC during unit load variation.

Simulation condition: initial MST and valve position of attemperator is $600{ }^{\circ} \mathrm{C}$ and $29.4 \%$, the on-site operating data of unit load $W$ and steam temperature of attemperator inlet $T i$, which is produced at the period of increasing unit load from $500 \mathrm{MW}$ to $950 \mathrm{MW}$, is utilized as the input variables of the simulation system, shown as Figure 8.

Four control strategies are employed in this control simulation.

1. the proposed OFAERMPC;

2. incremental model predictive controller (IMPC) based on the nominal model of the identified zonotope (29) with weighting coefficients $L_{x}=1$ and $L_{u}=0.01$, control horizon 5, prediction horizon 500 and sampling interval $T s=5 \mathrm{~s}$;

3. digital PI controller with proportional coefficient 5.26 and integral time 292.22 (design by matlab PID controller tuning modular);

4. standard AEMPC.

The control simulation results are shown in Figures 9 and 10, and control performance is listed in Table 4, where the performance index is mean square of the dynamic error. OFAERMPC has achieved 
the best performance index, and fluctuation of MST is the smallest among four controllers. Standard AEMPC obtains a satisfactory dynamic error, however, when dynamic process approaches the steady state (7000-1000 s), owing to the set point of control variable being unknown, there exists steady-state deviation that is unacceptable for control application. Both PI and IMPC can realize the offset-free control for MST system. The control action of IMPC is faster and lack of robust stability guarantee, we can find from Figures 9 and 10 that the attemperator valve position of IMPC varies sharply, causing strong fluctuations of MST, especially when the regulation approaches the steady state. Since the variation of attemperator valve position is smooth, the poor cooling effect of the PI controller results in the largest dynamic deviation. It also indicates that all the four controllers can meet the requirement for control variable restraint. On control performance: OFAERMPC is the best, PI controller has the largest dynamic deviation and IMPC shows a poor stability when process approaches the steady state, standard AEMPC is unfit for MST regulation system design. From the perspective of online computational burden: the total simulation time is shown in Table 4, the one of PI controller is the least, and OFAERMPC is better than IMPC and standard AEMPC. In summary, the control simulation illustrates the proposed OFAERMPC achieves a satisfied control performance for a unit load variation, an excellent applicability for real-time control is demonstrated because its online simulation time is a little more than that of PI and a stronger robustness is shown.

Table 4. Four control methods performance in Case 1.

\begin{tabular}{ccccc}
\hline & PI & IMPC & OLRMPC & OFAERMPC \\
\hline Performance index & 10.04 & 9.32 & 8.80 & 8.10 \\
Total Simulation time & 0.24 & 11.94 & 8.74 & 0.96 \\
\hline
\end{tabular}
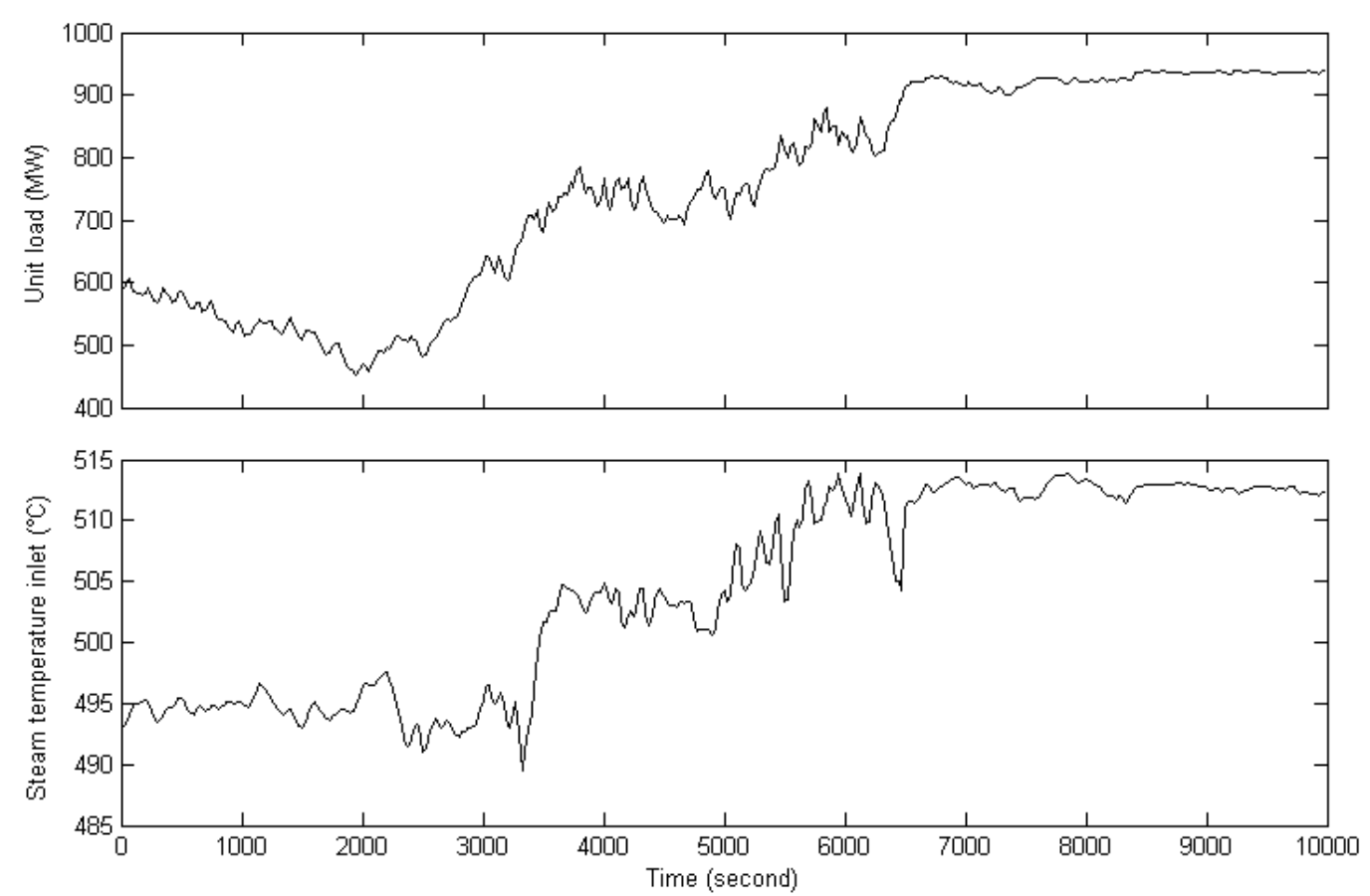

Figure 8. On-site operating data $W$ of and Ti for a 500 MW-950 MW unit load variation. 


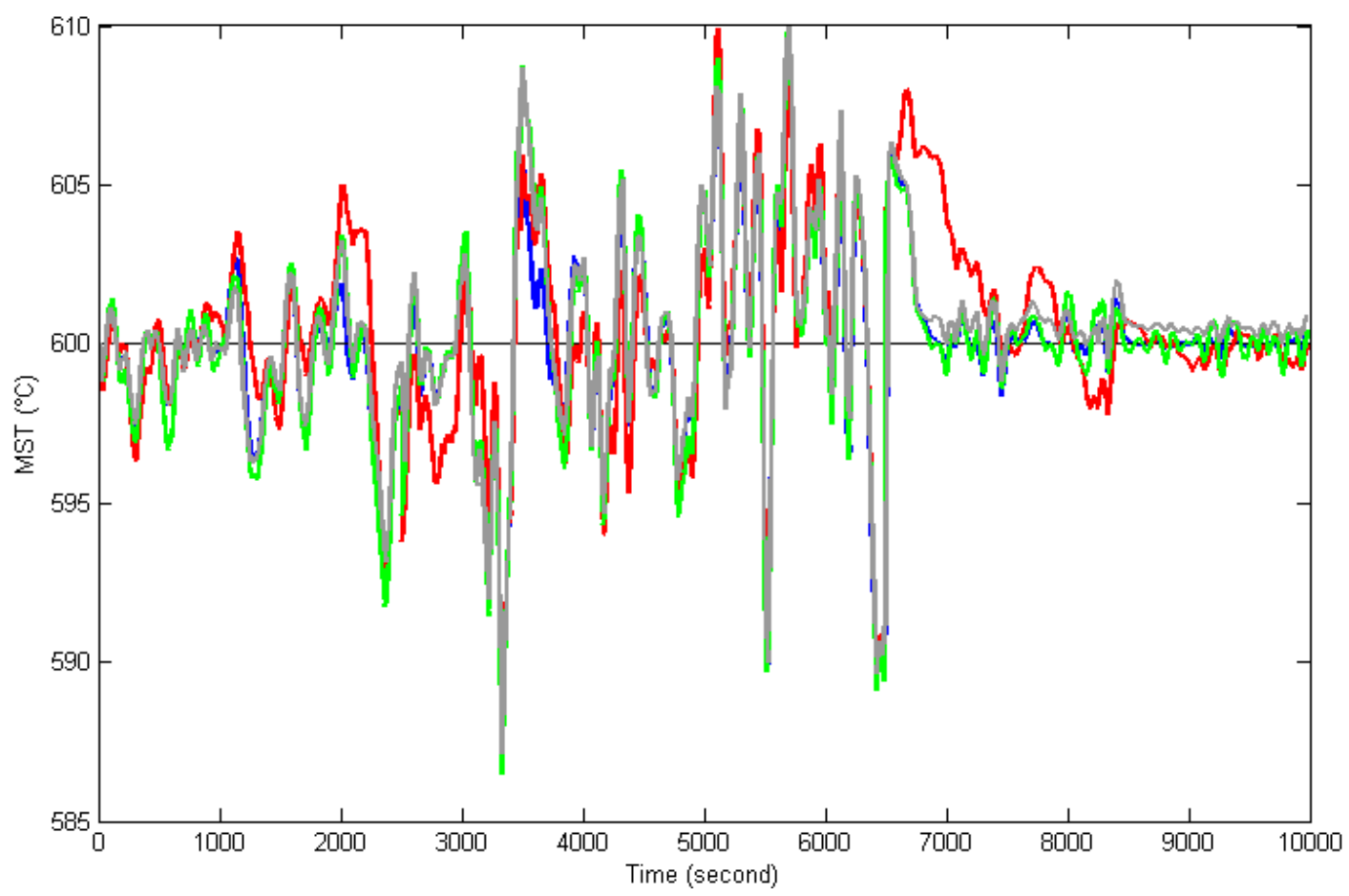

Figure 9. MST for a $500 \mathrm{MW}-950 \mathrm{MW}$ unit load variation (blue line: OFAERMPC; green line: incremental model predictive controller (IMPC); red line: PI; gray line: standard AEMPC; black line: set point of MST).

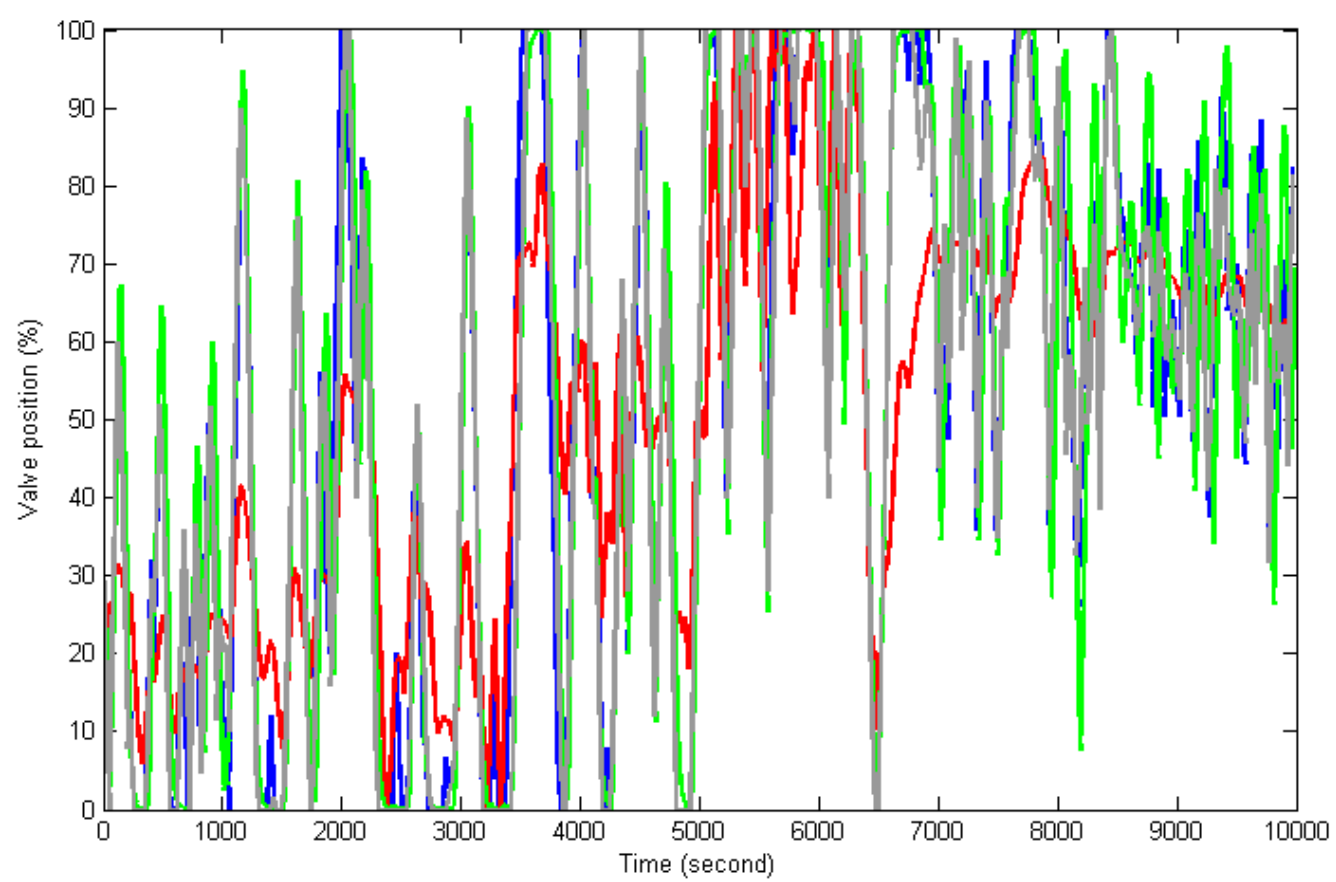

Figure 10. Valve position of MST for a 500 MW-950 MW unit load variation (blue line: OFAERMPC; green line: IMPC; red line: PI; gray line: standard AEMPC).

\section{Case 2. Unpredictable disturbance occurs}

Since complicated coal combustion process is involved in the boiler, unpredictable combustion status may change arising from coal sources variation, the heat exchange is thus disturbed. The steam temperature of attemperator inlet Ti and dynamic property of MST system will change when 
unmeasurable disturbance exists, the valve position of attemperator $u$ must be regulated to hold MST constant with less fluctuation. Objective of this control simulation is to test disturbance rejection performance of the proposed OFAERMPC.

Simulation condition: initial MST and valve position of attemperator is $600{ }^{\circ} \mathrm{C}$ and $75.5 \%$, at $t=0 \mathrm{~s}$ unpredictable disturbance occurs that results in steam temperature of attemperator inlet $T i$ varying frequently, the on-site operating data of unit load $W$ and $T i$ are used as the input variables of the simulation system, shown as Figure 11.

In this control simulation, OFAERMPC, PI and IMPC control strategies are employed, the corresponding control parameters are set the same as those in Case 1 The control simulation results are shown in Figures 12 and 13 and Table 5. It can be seen that control action of IMPC is faster and PI is slower compared with OFAERMPC; the dynamic deviation of OFAERMPC is the lowest; the dynamic deviation of IMPC is medium, variation of MST fluctuates sharply, oscillation occurs when process approaching the steady state; the variation of PI controller output is smooth and dynamic deviation is the largest. All of PI, IMPC and OFAERMPC can meet the requirement for control variable restraint when unpredictable disturbance occurs. On control performance: OFAERMPC is the best, PI controller achieves the largest dynamic deviation and a smooth control result, the dynamic deviation is satisfied for IMPC controller, but a poor stability is obtained when process approaches the steady state. From the perspective of online computational burden: the required calculation time of OFAERMPC is larger than that of the PI controller, while much less than the one of IMPC. In summary, this control simulation illustrates the proposed OFAERMPC achieves a satisfied control performance and applicability for real-time control when unpredictable disturbance occurs.

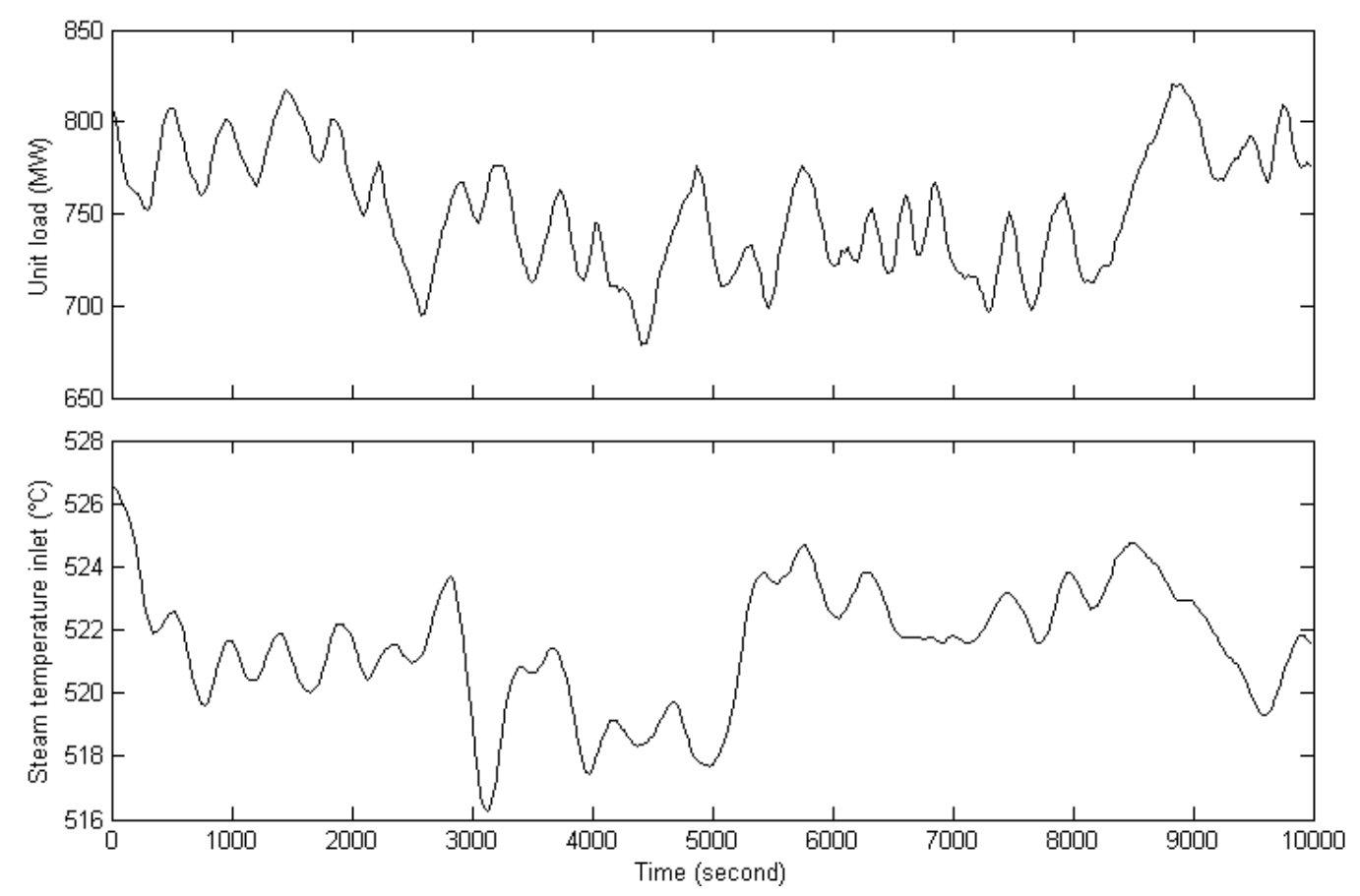

Figure 11. On-site operating data $W$ of and Ti when unpredictable disturbance occurs.

Table 5. Three control methods' performance in Case 2.

\begin{tabular}{cccc}
\hline & PI & IMPC & OFAERMPC \\
\hline Performance index & 5.05 & 0.71 & 0.55 \\
Total Simulation time & 0.22 & 0.05 & 0.98 \\
\hline
\end{tabular}




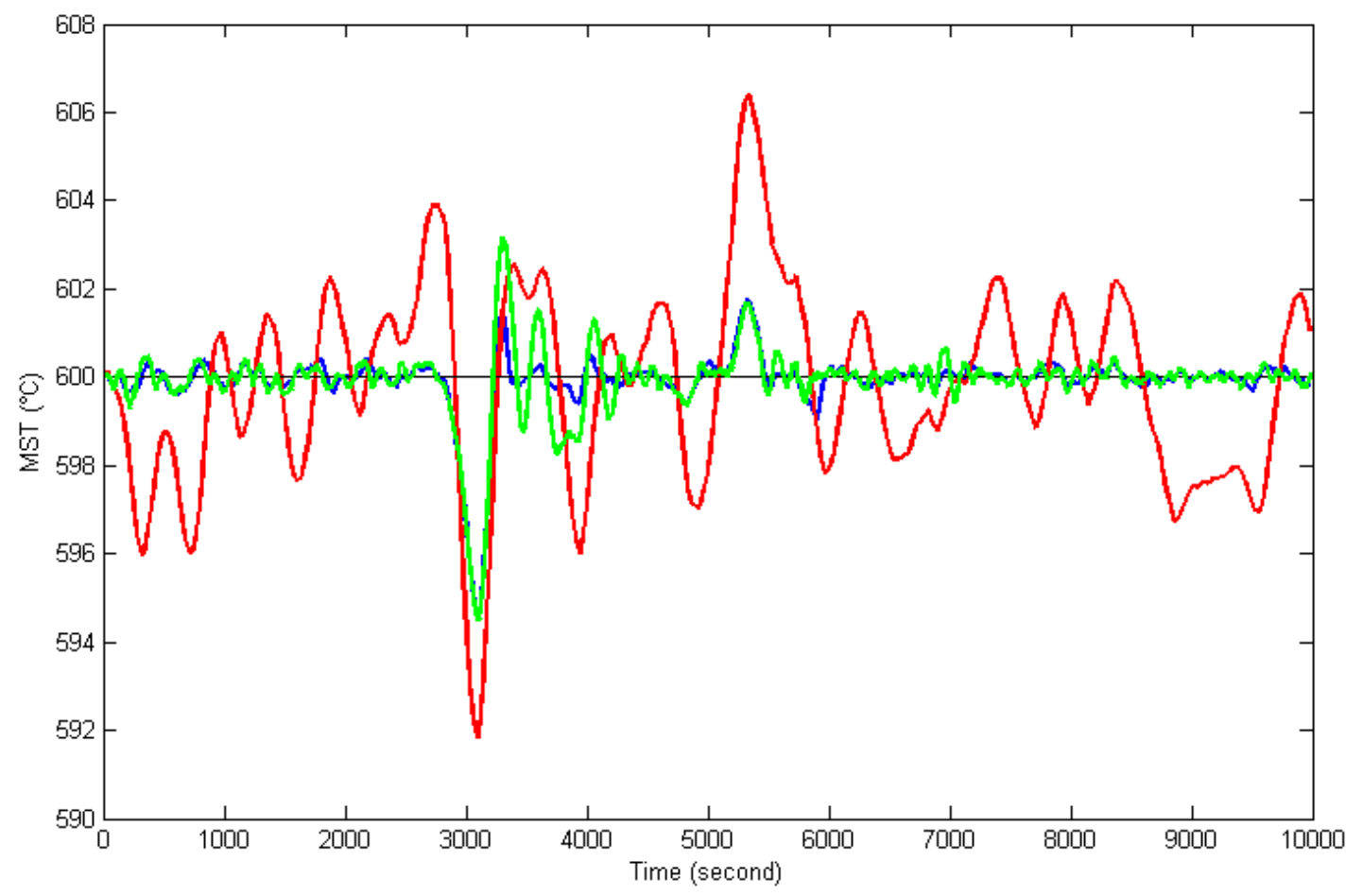

Figure 12. MST when unpredictable disturbance occurs (blue line: OFAERMPC; green line: IMPC; red line: PI; black line: set point of MST).

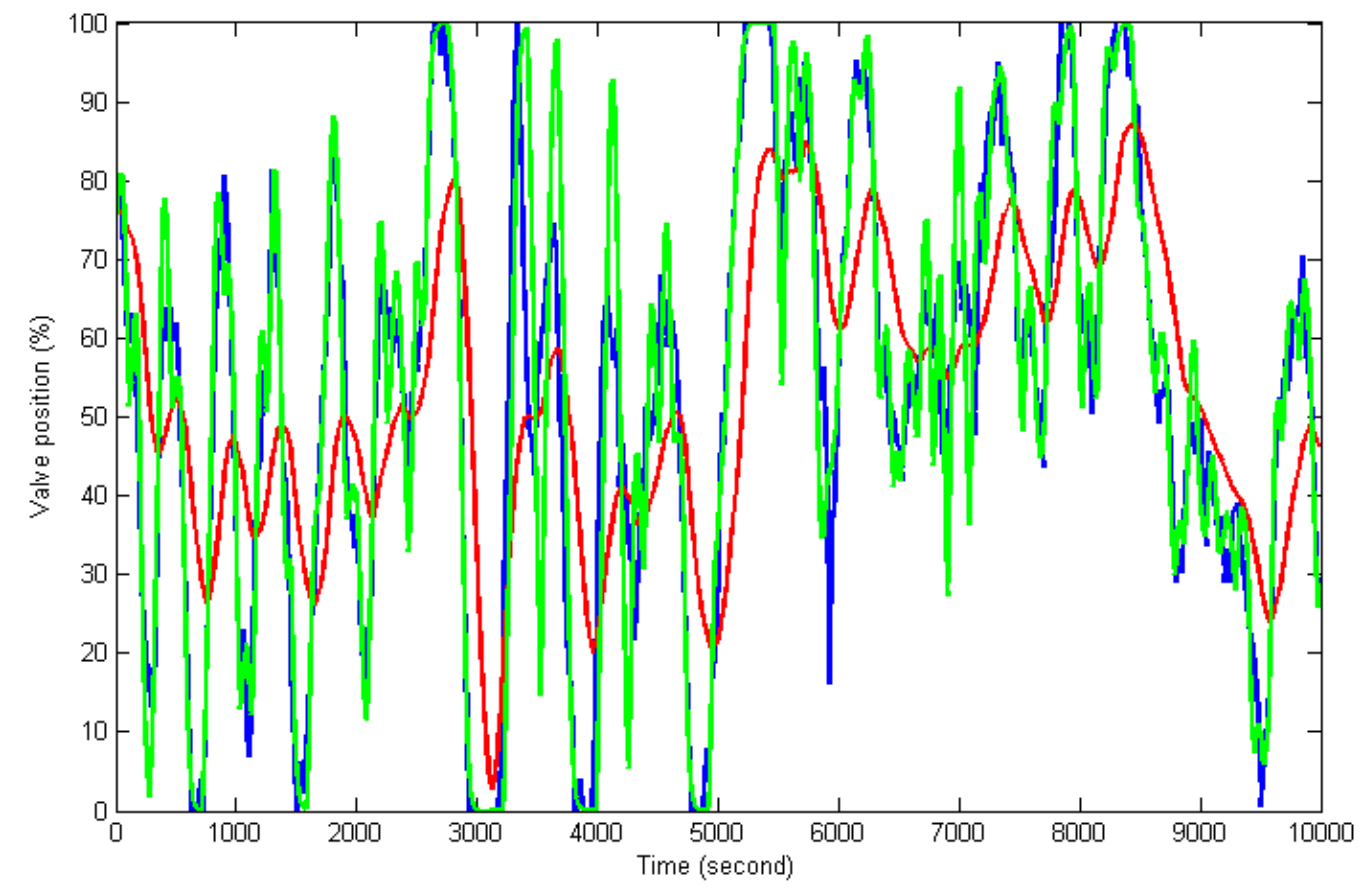

Figure 13. MST when unpredictable disturbance occurs (blue line: OFAERMPC; green line: IMPC; red line: PI).

\section{Case 3. Plant behavior changes}

The real dynamic property of MST system may deviate from the designed one due to aging of equipment, which may cause performance degradation of the control system. The objective of this control simulation is to test the control performance of OFAERMPC strategy when an unknown change of dynamic property of controlled system occurs. 
Simulation condition: initial MST and valve position of attemperator is $600{ }^{\circ} \mathrm{C}$ and $75.5 \%$, at $t=2000 \mathrm{~s}$ unpredictable disturbance occurs, the on-site operating data of unit load $W$ and $\mathrm{Ti}$ are used as the input variables of the simulation system, shown as Figure 11.

OFAERMPC, PI and IMPC control strategies are employed in this simulation, the corresponding control parameters are set the same as those in Case 1.

The control simulation results are shown in Figures 14 and 15 and Table 6. They indicate that control performance of all the three control strategies get worse in case of plant behavior change. Among them, the performance degradation of OFAERMPC is smaller than that of PI after $t=2000 \mathrm{~s}$. Compared with the lines in Figures 12 and 13, due to the restriction of control action getting worse of OFAERMPC regulation is more serious than that of PI regulation when $t=5000-6000 \mathrm{~s}$ and $t=7500-8500 \mathrm{~s}$, the robustness of OFAERMPC is better for the other time; the trend of getting worse for IMPC is obvious, and oscillation aggravates when the process approaches the steady state. PI, IMPC and OFAERMPC can meet the requirement for control variable restraint when dynamic property of MST system changes. Regarding the control performance, OFAERMPC has the similar robustness to PI, and robustness of IMPC is the poorest. From the perspective of online computational burden: the required calculation time of OFAERMPC is larger than that of the PI controller, but much less than that of IMPC. In summary, this control simulation illustrates the proposed OFAERMPC achieves a satisfied control performance and applicability for real-time control when strong plant behavior change occurs.

Table 6. Three control methods performance after an unknown behavior change in Case 3.

\begin{tabular}{cccc}
\hline & PI & IMPC & OFAERMPC \\
\hline Performance index (unchanged behavior) & 5.36 & 0.88 & 0.68 \\
Performance index (changed behavior) & 7.17 & 3.32 & 2.28 \\
Total Simulation time & 0.23 & 10.36 & 0.98 \\
\hline
\end{tabular}

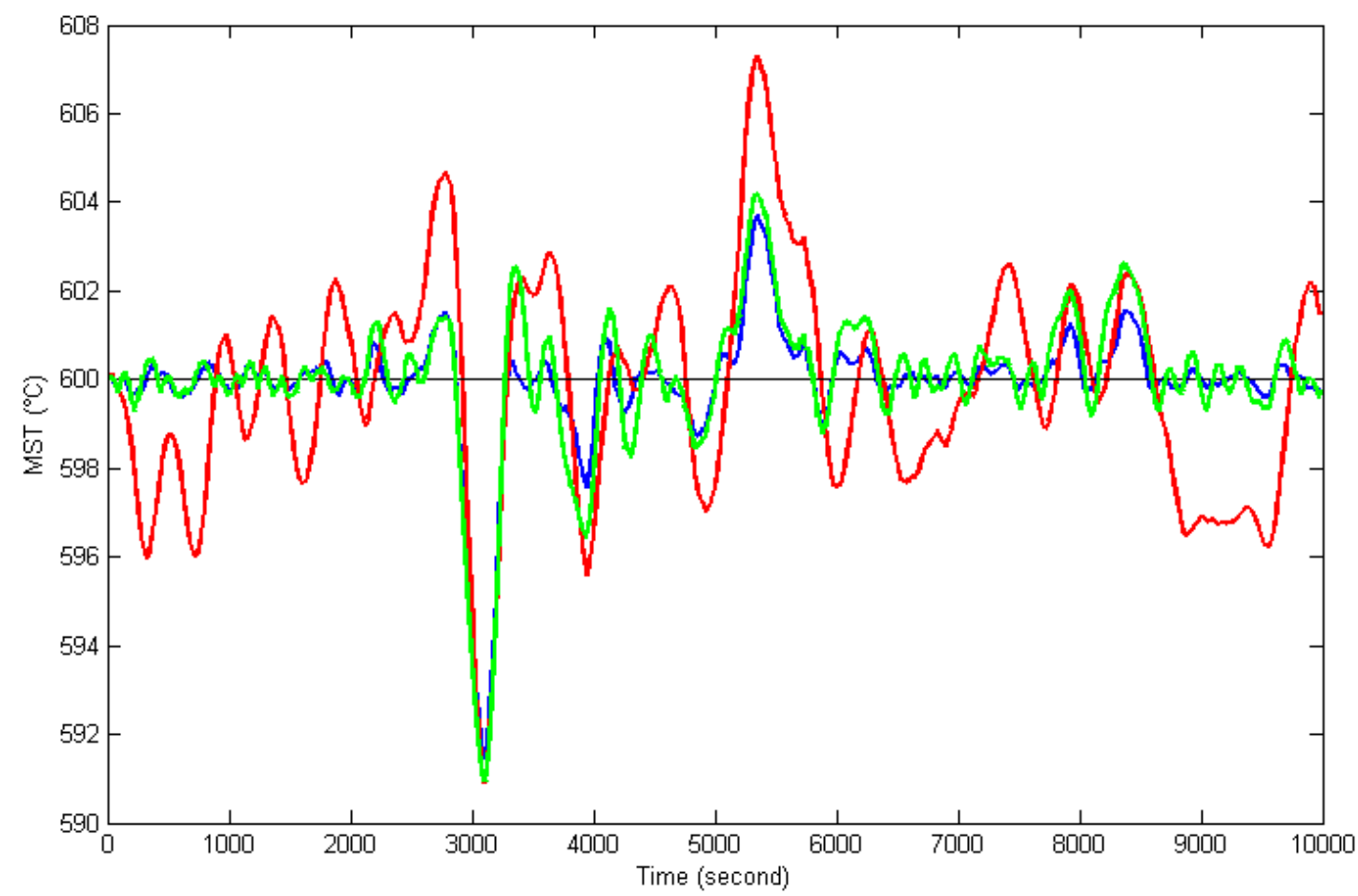

Figure 14. MST for an unknown change of dynamic property of controlled system (blue line: OFAERMPC; green line: IMPC; red line: PI; black line: set point of MST). 


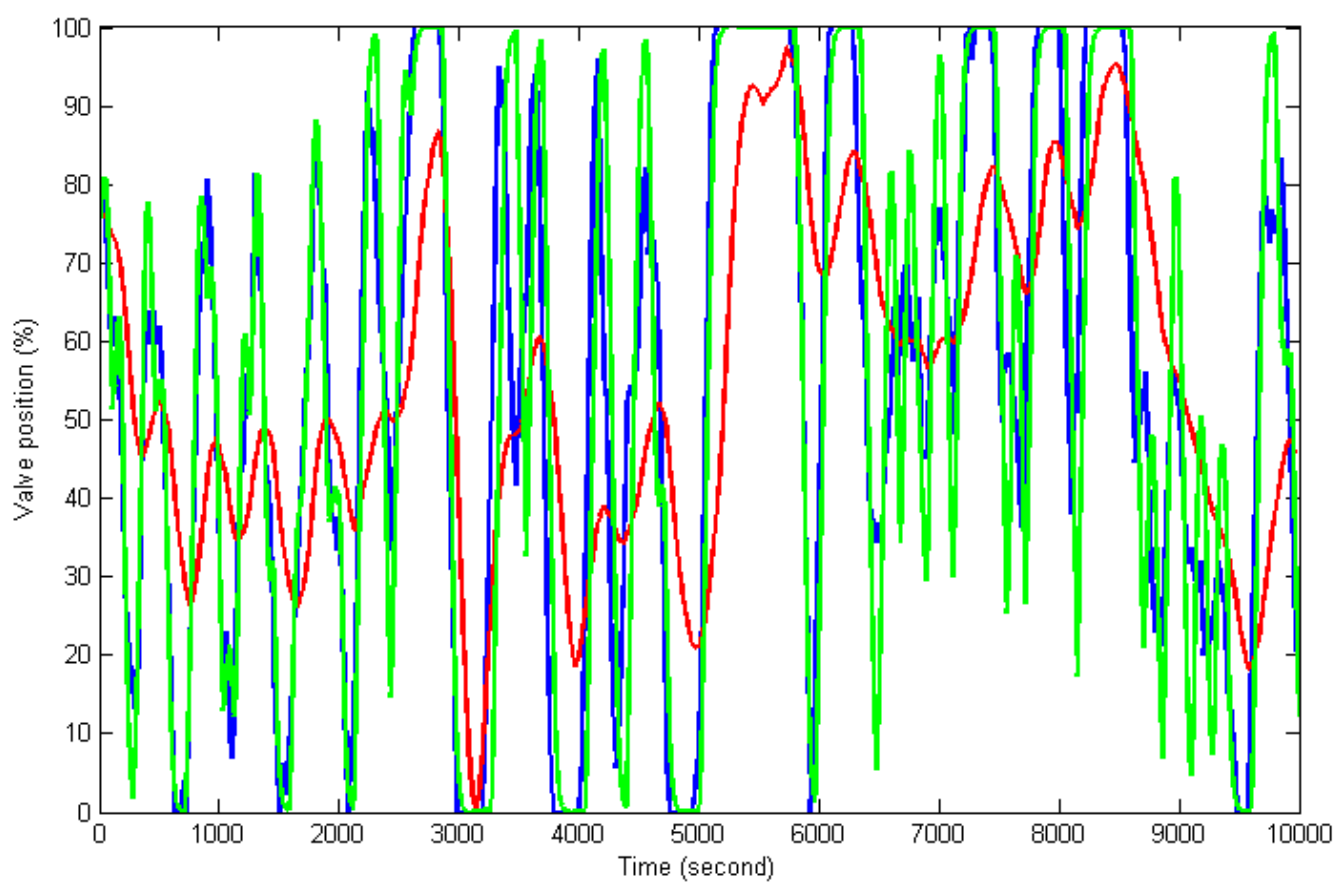

Figure 15. Valve position for an unknown change of dynamic property of controlled system (blue line: OFAERMPC; green line: IMPC; red line: PI).

\section{Conclusions}

Due to large thermal inertia, high nonlinearity and various unpredictable disturbances, the MST system is challenging to regulate with guaranteeing robust stability and less online computational effort. This paper presents a novel offline RMPC approach based on zonotope-type uncertain model, which acquires the following contributions:

1. In the offline design stage, an explicit RMPC control law design method with improved convergence is proposed by introducing two extra parameters;

2. Based on the nominal model of zonotope, a manipulated variable target observer is developed to make control results no offset exists.

To demonstrate the effectiveness of the proposed OFAERMPC, three control simulations of MST system are conducted. Compared with standard AEMPC, the computation burden in offline design stage of OFAERMPC largely reduces with a simpler subspace partition. In addition, as for control performance, the proposed OFAERMPC achieves the least dynamic deviation, and provides satisfied robustness with less online computational burden, which is useful to overcome the most urgent concern in thermal process control practice.

Author Contributions: Conceptualization, D.W.; methodology, D.W. and X.W.; validation, D.W. and J.S.; formal analysis, D.W.; investigation, D.W.; resources, D.W.; data curation, D.W.; writing—original draft preparation, D.W.; writing - review and editing, X.W.; visualization, D.W.; supervision, J.S.; project administration, J.S.; funding acquisition, J.S. and X.W. All authors have read and agreed to the published version of the manuscript.

Funding: This research was funded by National Natural Science Foundation of China (NSFC) under Grants 51936003 and the Royal Society-Sino British Fellowship Trust International Fellowship.

Conflicts of Interest: The authors declare no conflict of interest. 


\section{Abbreviations}

The following abbreviations are used in this manuscript:

$\begin{array}{ll}\text { AEMPC } & \text { Approximated explicit model predictive control } \\ \text { CFPP } & \text { Coal-fired power plant } \\ \text { MIMO } & \text { Multiple inputs multiple outputs } \\ \text { MPC } & \text { Model predictive control } \\ \text { MST } & \text { Main steam temperature } \\ \text { NN } & \text { Neural network } \\ \text { OFAERMPC } & \text { Offset-free approximated explicit robust model predictive control } \\ \text { PWA } & \text { Piecewise affine } \\ \text { PID } & \text { Proportion integration differentiation } \\ \text { RMPC } & \text { Robust model predictive control } \\ \text { SISO } & \text { Single input single output } \\ \text { SMI } & \text { Set membership identification }\end{array}$

\section{References}

1. China Electricity Council. Letters of Chinese Electricity Power Statistics in 2018; China National Energy Administration: Beijing, China, 2018. (In Chinese)

2. Wu, X.; Shen, J.; Li, Y.; Lee, K.Y. Fuzzy modeling and predictive control of superheater steam temperature for power plant. ISA Trans. 2015, 56, 241-251. [CrossRef] [PubMed]

3. Song, X.; Liu, C.; Song, Z.; Song, X. Robust PID control for Steam superheater. In Proceedings of the Third International Conference on Machine Learning and Cybernetics, Shanghai, China, 26-29 August 2004; pp. 988-991.

4. Kim, H.; Kim, E.; Kim, J.; Lee, K.; Kim, S.; Han, Y. Prediction-based feedforward control of superheated steam temperature of a power plant. Electr. Power Energy Syst. 2015, 71, 351-257. [CrossRef]

5. Hlava, J.; Opalka, J.; Johansen, A. Model predictive control of power plant superheater - comparison of multi model and nonlinear approaches. In Proceedings of the 2013 18th International Conference on Methods in Automation \& Robotics, Miedzyzdroje, Poland, 27-29 August 2013; pp. 311-316.

6. Wang, G.; Yan, W.; Chen, S.; Zhang, X.; Shao, H. Multivariable constrained predictive control of main steam temperature in ultra-supercritical coal-fired power unit. J. Energy Inst. 2015, 88, 181-187. [CrossRef]

7. Liang, W.; Wang, W.; Zhang, X. Fuzzy-PID control system of main steam temperature based on intermediate point temperature feedforward. Electr. Power Sci. Eng. 2019, 35, 72-78.

8. Valsalam, R.; Snish, S.; Singh, R. Boiler Modelling and Optimal Control of Steam Temperature in Power Plants. IFAC Proc. Vol. 2009, 42, 125-130. [CrossRef]

9. Ma, L.; Lin, Y.; Lee, K.Y. Superheater steam temperature control for a $300 \mathrm{MW}$ boiler unit with inverse dynamic process models. In Proceedings of the IEEE PES General Meeting, Providence, RI, USA, 25-29 July 2010; pp. 1-6.

10. Ma, L.; Lee, K.Y.; Ge, Y. An improved predictive optimal controller with elastic search space for steam temperature control of large-scale supercritical power unit. In Proceedings of the 51st IEEE Conference on Decision and Control, Maui, HI, USA, 10-13 December 2012; pp. 2024-2029.

11. Mayne, D.Q.; Rawlings, J.B.; Rao, C.V.; Scokaert, P.O. Constrained model predictive control: Stability and optimality. Automatica 2000, 36, 789-814. [CrossRef]

12. Mayne, D.Q. Model predictive control: Recent developments and future promise. Automatica 2014, 50, 2967-2986. [CrossRef]

13. Hu, H.; Zhang, J.; Yang, Q. Dynamic matrix control for main steam temperature control system based on optimal state estimation. Control Decis. 2019, 34, 1475-1480.

14. Hlava, J. Model predictive control of the superheater temperature based on a piecewise affine model. In Proceedings of the UKACC International Conference on Control 2010, Coventry, UK, 7-10 September 2010; pp. 423-428. 
15. Zhao, H.; Shen, J.; Shen, D.; Li, Y. Multiple model disturbance rejection predictive control of main steam temperature. Proc. CSEE 2014, 32, 5763-5770.

16. Van Overschee, P.; De Moor, B. N4SID: Subspace algorithms for the identification of combined deterministic stochastic systems. Automatica 1994, 30, 75-93. [CrossRef]

17. Vuolo, F.; Ng, W.; Atzberger, C. Smoothing and gap-filling of high resolution muti-spectral time series: Example of Landsat data. Int. J. Appl. Earth Obs. Geoinf. 2017, 57, 202-213. [CrossRef]

18. Kim, J.; Ryu, J. A heuristic gap filling method for daily precipition series. Water Recour Manag. 2016, 30, 2275-2294. [CrossRef]

19. Shary, S. Maximum consistency method for data fitting under interval uncertainty. J. Glob. Optim. 2016, 66, 111-126. [CrossRef]

20. Duan, Y.; Wang, Q.; Liao, Y.; Wei, L. Exploration on fitting function in data processing of physical chemistry experiments. J. Cap. Norm. Univ. 2019, 40, 33-36.

21. Vera-Sanchez, J.; Ruiz-Morales, C.; Gonzalez-Lopez, A. Monte Carlo uncertainty analysis of dose estimates in radiochromic film dosimetry with single-channel and multichannel algorithms. Phys. Medica 2018, 47, $23-33$. [CrossRef]

22. Castro, E.; Ahnert, C.; Buss, O.; Garacia-Herranz, N.; Hoefer, A.; Porsch, D. Improving PWR core simulations by Monte Carlo uncertainty analysis and Bayesian inference. Ann. Nucl. Energy 2016, 95, 148-156. [CrossRef]

23. Zhang, J.; Li, Y.; Huang, G.; Baetz, B.; Liu, J. Uncertainty analysis for effluent trading planning using a Bayesian estimation-based simulation modelling approach. Water Res. 2017, 116, 159-181. [CrossRef]

24. Liu, J.; Zeng, X.; Wu, J.; Liang, X.; Sun, Y.; Zhan, H. Assessing titanium dioxide nanoparticles transport models by Bayesian uncertainty analysis. Stoch. Environ. Res. Risk Assess. 2018, 32, 3365-3379. [CrossRef]

25. Milanese, M.; Taragna, M. H infinity set membership identification: A survey. Atomatica 2005, 41, $2019-2032$. [CrossRef]

26. Cerone, V.; Piga, D.; Regruto, D. Set-membership error-in-variables identification through convex relaxation techniques. IEEE Trans. Autom. Control 2012, 57, 517-522. [CrossRef]

27. Zhou, B.; Qian, K.; Ma, X.; Dai, X. Ellipsoidal bounding set-membership identification approach for robust fault diagnosis with application to mobile robots. J. Syst. Eng. Electron. 2017, 28, 986-995. [CrossRef]

28. Reppa, V.; Tzse, A. Fault diagnosis based on set membership identification using output-error models. Int. J. Adapt. Control Signal Process. 2016, 30, 224-255. [CrossRef]

29. Scott, J.; Raimaondo, D.; Marseglia, G.; Braatz, R. Constrained zonotopes: A new tool for set-based estimation and fault detection. Automatica 2016, 69, 126-136. [CrossRef]

30. Alamo, T.; Bravo, J.; Camacho, E. Guaranteed state estimation by zonotopes. Automatica 2006, 41, $1035-1043$. [CrossRef]

31. Wang, D.; Wu, X.; Pan, L.; Shen, J.; Lee, K.Y. A novel zonotope-based set-membership identification approach for uncertain system. In Proceedings of the 2017 IEEE Conference on Control Technology and Applications, Kohala Coast, HI, USA, 27-30 August 2017; pp. 1420-1425.

32. Kothare, M.V.; Balakrishnan, V.; Morari, M. Robust constrained model predictive control using linear matrix inequalities. Automatica 1996, 32, 1361-1379. [CrossRef]

33. Sartipizzadeh, H.; Vincent, T. A new robust MPC using an approximate convex hull. Automatica 2018, 92, 115-122. [CrossRef]

34. Bemporad, A.; Filippi, C. An algorithm for approximate multiparametric convex programming. Comput. Optim. Appl. 2006, 35, 87-108. [CrossRef]

35. Pluymers, B.; Suykens, J.A.; Moor, B.D. Min-max feedback MPC using a time-varying terminal constraint set and comments on "Efficient robust constrained model predictive control with a time-varying terminal constraint set". Syst. Control Lett. 2005, 54, 1143-1148. [CrossRef]

36. Wu, Q.; Xi, Y.; Nagy, Z.; Li, D. A real-time optimization framework for the time-varying economic environment. Comput. Chem. Eng. 2018, 115, 333-341. [CrossRef]

37. Feng, L.; Alliez, P.; Buse, L.; Delingette, H.; Desbrun, M. Curved optimal Delaunay triangulation. Acm Trans. Graph. 2018, 37, 61.1-61.16. [CrossRef]

38. Zhu, Y.; Butoyi, F. Case studies on closed-loop identification for MPC. Control Eng. Pract. 2002, 10, 404-417. [CrossRef] 
39. Soderstrom, T.; Stoica, P. System Identification; Prentice-Hall: London, UK, 1989.

40. Lofberg, J. Yalmip: A toolbox for modelling and optimization in Matlab. In Proceedings of the 2004 IEEE International Conference on Robotics and Automation, New Orleans, LA, USA, 2-4 September 2004; pp. 284-289.

(C) 2020 by the authors. Licensee MDPI, Basel, Switzerland. This article is an open access article distributed under the terms and conditions of the Creative Commons Attribution (CC BY) license (http:// creativecommons.org/licenses/by/4.0/). 